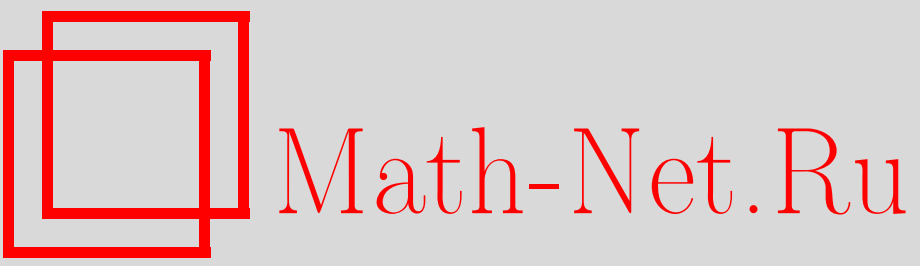

И. Г. Шевцова, О точности нормальной аппроксимации для обобщенных пуассоновских распределений, Теория вероятн. и ее примен., 2013, том 58, выпуск $1,152-176$

DOI: https://doi.org/10.4213/tvp4498

Использование Общероссийского математического портала Math-Net.Ru подразумевает, что вы прочитали и согласны с пользовательским соглашением

http: //www . mathnet.ru/rus/agreement

Параметры загрузки :

IP : 3.89 .185 .249

26 апреля 2023 г., 12:10:53 
ТЕОРИЯ ВЕРОЯТНОСТЕЙ

Том 58

И ЕЕ ПРИМЕНЕНИЯ

Выпуск 1

2013

(C) 2013 г.

ШЕВЦОВА И. Г.

\section{О ТОЧНОСТИ НОРМАЛЬНОЙ АППРОКСИМАЦИИ ДЛЯ ОБОБЩЕННЫХ ПУАССОНОВСКИХ РАСПРЕДЕЛЕНИЙ ${ }^{1)}$}

Впервые найдено точное значение асимптотически правильной
константы в аналоге неравенства Берри-Эссеена для пуассонов-
ских случайных сумм независимых одинаково распределенных слу-
чайных величин $X_{1}, X_{2}, \ldots$, имеющих моменты третьего порядка.
Кроме того, для равномерного расстояния $\Delta_{\lambda}$ между функций рас-
пределения стандартного нормального закона и функцией распре-
деления центрированной и нормированной случайной суммы $S_{\lambda}=$
$X_{1}+\cdots+X_{N_{\lambda}}$, где $N_{\lambda}$ имеет распределение Пуассона с параметром
$\lambda>0$ и независима от $X_{1}, X_{2}, \ldots$, получены оценки:
$\Delta_{\lambda} \leqslant \frac{2 \ell_{\lambda}}{3 \sqrt{2 \pi}}+0.5 \cdot \ell_{\lambda}^{2}<0.2660 \cdot \ell_{\lambda}+0.5 \cdot \ell_{\lambda}^{2}, \quad$ где $\ell_{\lambda}=\frac{\mathbf{E}\left|X_{1}\right|^{3}}{\sqrt{\lambda}\left(\mathbf{E} X_{1}^{2}\right)^{3 / 2}}$

Показано, что эта оценка неулучшаема в отношении множителя $2 /(3 \sqrt{2 \pi})=0.2659 \ldots$ при $\ell_{\lambda}$. Для случая, когда распределение $X_{1}$ симметрично, доказана уточненная оценка

$$
\Delta_{\lambda} \leqslant \frac{1+2 \varkappa}{2 \sqrt{2 \pi}} \ell_{\lambda}+0.4 \cdot \ell_{\lambda}^{2}<0.2391 \cdot \ell_{\lambda}+0.4 \cdot \ell_{\lambda}^{2},
$$

где $\varkappa=\sup _{x>0}\left(\cos x-1+x^{2} / 2\right) / x^{3}=0.0991 \ldots$ Показано, что значение множителя при $\ell_{\lambda}$ в этой оценке не может быть меньше $(2 \sqrt{2 \pi})^{-1}=0.1994 \ldots$. Получены аналогичные оценки при ослабленных моментных условиях, когда $\mathbf{E}\left|X_{1}\right|^{2+\delta}<\infty$ с некоторым $0<\delta<1$.

Ключевые слова и фразы: пуассоновская случайная сумма, центральная предельная теорема, оценка скорости сходимости, нормальная аппроксимация, неравенство Берри-Эссеена, асимптотически правильная константа.

\footnotetext{
* Московский государственный университет им. М. В. Ломоносова, факультет вычислительной математики и кибернетики; Институт проблем информатики РАН, Москва, Россия; e-mail: ishevtsova@cs.msu.su

1) Работа выполнена при поддержке РФФИ (проекты 11-01-00515a, 11-07-00112a, 11-01-12026-офи-м) и Министерства образования и науки (грант МK-2256.2012.1, госконтракт 16.740.11.0133).
} 
1. Введение. Пусть $X, X_{1}, X_{2}, \ldots$ - независимые одинаково распределенные случайные величины (н.о.р.с.в.) с общей функцией распределения $F(x)=\mathbf{P}(X<x), x \in \mathbf{R}$. Предположим, что

$$
\mathbf{E} X^{2}>0, \quad \beta_{2+\delta} \equiv \mathbf{E}|X|^{2+\delta}<\infty
$$

при некотором $\delta \in[0,1]$. Множество всех функций распределения случайных величин $X$, удовлетворяющих указанному условию, обозначим $\mathscr{F}_{2+\delta}$. Пусть $F_{2+\delta, \mathrm{s}}-$ множество всех симметричных функций распределения из $\mathscr{F}_{2+\delta}$. Всюду далее под записью $X \in \mathscr{F}_{2+\delta}\left(X \in \mathscr{F}_{2+\delta, \mathrm{s}}\right)$ подразумеваем, что функция распределения $F$ случайной величины $X$ принадлежит классу $\mathscr{F}_{2+\delta}\left(\mathscr{F}_{2+\delta, \mathrm{s}}\right)$, также будем отождествлять и записи функционалов, зависящих от распределения случайной величины $X$, в качестве аргументов которых указана функция распределения $F$ случайной величины $X$ или сама случайная величина $X$. Например, $\beta_{2+\delta}=$ $\beta_{2+\delta}(F)=\beta_{2+\delta}(X)=\mathbf{E}|X|^{2+\delta}$.

Пусть $N_{\lambda}$ - случайная величина, имеющая распределение Пуассона с параметром $\lambda>0$ :

$$
\mathbf{P}\left(N_{\lambda}=k\right)=\frac{\lambda^{k} e^{-\lambda}}{k !}, \quad k=0,1,2, \ldots,
$$

и такая, что на расширенном вероятностном пространстве при каждом $\lambda>0$ случайные величины $N_{\lambda}, X_{1}, X_{2}, \ldots$ независимы. Случайная величина $S_{\lambda}=X_{1}+\cdots+X_{N_{\lambda}}$ называется пуассоновской случайной суммой (для определенности полагаем $\sum_{k=1}^{0}(\cdot)=0$ ), а ее распределение - обобщенным пуассоновским. Пуассоновские случайные суммы $S_{\lambda}$ являются весьма популярными математическими моделями многих реальных объектов. В частности, в страховой математике случайная величина $S_{\lambda}$ описывает суммарное страховое требование в классическом процессе риска в «динамическом» случае. Многие примеры прикладных задач из самых разнообразных областей, в которых используются пуассоновские случайные суммы, приведены, например, в книгах [26], [21].

Как известно, для $F \in \mathscr{F}_{2}$ распределения пуассоновских случайных сумм $S_{\lambda}$ асимптотически нормальны, что обусловливает большую важность задачи изучения точности нормальной аппроксимации для распределений пуассоновских случайных сумм.

Несложно убедиться, что $\mathbf{E} S_{\lambda}=\lambda \mathbf{E} X, \mathbf{D} S_{\lambda}=\lambda \mathbf{E} X^{2}$. Функцию распределения стандартизованной пуассоновской случайной суммы

$$
\widetilde{S}_{\lambda}=\frac{S_{\lambda}-\mathbf{E} S_{\lambda}}{\sqrt{\mathbf{D} S_{\lambda}}}=\frac{S_{\lambda}-\lambda \mathbf{E} X}{\sqrt{\lambda \mathbf{E} X^{2}}}
$$

обозначим $F_{\lambda}(x)$. Для $F \in \mathscr{F}_{2+\delta}$ с $0<\delta \leqslant 1$ также введем следующие обозначения:

$$
L_{0}=L_{0}(F)=L_{0}(X)=\frac{\mathbf{E}|X-\mathbf{E} X|^{2+\delta}}{(\mathbf{D} X)^{1+\delta / 2}},
$$




$$
\begin{aligned}
& L_{1}=L_{1}(F)=L_{1}(X)=\frac{\mathbf{E}|X|^{2+\delta}}{\left(\mathbf{E} X^{2}\right)^{1+\delta / 2}}, \\
& \ell_{\lambda}=\ell_{\lambda}(F)=\frac{L_{1}(F)}{\lambda^{\delta / 2}}=\frac{\mathbf{E}|X|^{2+\delta}}{\lambda^{\delta / 2}\left(\mathbf{E} X^{2}\right)^{1+\delta / 2}} .
\end{aligned}
$$

При $\delta=0$ положим $L_{0}=L_{1}=\ell_{\lambda}=1$. Величины $L_{0}$ и $L_{1}$ будем называть соответственно центральным и нецентральным ляпуновскими отношениями, а $\ell_{\lambda}$ - нецентральной ляпуновской дробью. Пусть

$$
\begin{aligned}
\Phi(x) & =\frac{1}{\sqrt{2 \pi}} \int_{-\infty}^{x} e^{-t^{2} / 2} d t, \\
\Delta_{\lambda} & =\Delta_{\lambda}(F)=\Delta_{\lambda}(X)=\sup _{x}\left|F_{\lambda}(x)-\Phi(x)\right| .
\end{aligned}
$$

Утверждение об асимптотической нормальности обобщенных пуассоновских распределений означает, что равномерное расстояние $\Delta_{\lambda}(F)$ между допредельной функцией распределения стандартизованной пуассоновской случайной суммы и предельной нормальной функцией распределения стремится к нулю с ростом $\lambda$. Этот факт имеет место для любой функции распределения $F \in \mathscr{F}_{2}$. Однако условие принадлежности $F \in \mathscr{F}_{2}$ не является достаточным для конструирования разумных оценок скорости сходимости $\Delta_{\lambda}(F) \rightarrow 0$, что весьма важно для прикладных задач, в которых используется аппроксимация нормальным распределением. А именно, как будет показано в теореме 8 , для любой неотрицательной функции $\psi(\lambda)$ такой, что $\lim _{\lambda \rightarrow \infty} \psi(\lambda)=0$, найдется функция распределения $F \in \mathscr{F}_{2}$ такая, что $\Delta_{\lambda}(F) \geqslant \psi(\lambda)$ для всех достаточно больших $\lambda$. Кроме того, в теореме 5 будет продемонстрировано, что

$$
\limsup _{\lambda \rightarrow \infty} \sup _{F \in \mathscr{F}_{2}} \Delta_{\lambda}(F)>0.54006
$$

С другой стороны, если для некоторого $0<\delta \leqslant 1$ известно, что $F \in \mathscr{F}_{2+\delta}$, то скорость сходимости в центральной предельной теореме уже представляется возможным оценить в терминах соответствующих моментов при помощи неравенства типа Берри-Эссеена

$$
\Delta_{\lambda} \leqslant C_{\mathrm{BE}}(\delta) \frac{L_{1}}{\lambda^{\delta / 2}}=C_{\mathrm{BE}}(\delta) \ell_{\lambda}
$$

где $C_{\mathrm{BE}}(\delta)$ зависит только от $\delta$. Говоря об оценках типа Берри-Эссеена для пуассоновских сумм, нельзя, конечно же, не упомянуть их историю, краткое описание которой мы приведем, следуя линии изложения этого вопроса в книге [5].

Традиционно наибольшее внимание уделялось случаю $\delta=1$, поскольку, как можно убедиться, при $\delta>1$ скорость сходимости остается в общем случае такой же, как при $\delta=1$. При этом оценки точности нормальной аппроксимации для обобщенных пуассоновских распределений 
выписывались в терминах центральных ляпуновских отношений $L_{0}$ как следствия оценок для случайных сумм с произвольным асимптотически вырожденным индексом и по аналогии с оценками для сумм неслучайного числа независимых с.в. Такие оценки имели довольно громоздкий вид (см., например, [24], [7]).

Однако при конструировании оценок точности нормальной аппроксимации для обобщенных пуассоновских распределений более естественным оказалось использование нецентральных ляпуновских отношений, в терминах которых такие оценки принимают совсем простой вид (см. (1)). По-видимому, впервые неравенство (1) было доказано для $\delta=1$ в неопубликованной диссертации Г. В. Ротарь в 1972 г. [11] и опубликовано без доказательства в статье того же автора в 1976 г. [12] с $C_{\mathrm{BE}}(1)=2.23$. В 1983 г. с использованием традиционной техники, основанной на неравенстве сглаживания Эссеена, оценка (1) была доказана Р. фон Чосси и Г. Рапплом [23] с $C_{\mathrm{BE}}(1)=2.21$ (причем авторы этой работы в формулировке соответствующей теоремы объявили значение $C_{\mathrm{BE}}(1)=3$, что, конечно, верно, но фактически по ходу доказательства они получили значение $\left.C_{\mathrm{BE}}(1)=2.21\right)$.

Следующий прорыв в оценивании постоянной $C_{\mathrm{BE}}(1)$ был совершен в 1993 г. Р. Михелем [29] с использованием свойства безграничной делимости обобщенных пуассоновских распределений и верхних оценок константы $A(1)$ в классическом неравенстве Берри-Эссеена для сумм неслучайного числа н.о.р.с.в.:

$$
\sup _{x \in \mathbf{R}}\left|\mathbf{P}\left(\frac{X_{1}+\cdots+X_{n}-n \mathbf{E} X}{\sqrt{n \mathbf{D} X}}<x\right)-\Phi(x)\right| \leqslant A(\delta) \frac{L_{0}(X)}{n^{\delta / 2}},
$$

$n \geqslant 1, X \in \mathscr{F}_{2+\delta}$. Относительно константы $A(1)$ в настоящий момент известно, что

$$
0.4097 \ldots=\frac{\sqrt{10}+3}{6 \sqrt{2 \pi}} \leqslant A(1)<0.4748
$$

(нижняя оценка найдена К.-Г. Эссееном в [25], верхняя - автором в [31]). Наилучшие оценки констант $A(\delta)$ при $0<\delta<1$ приведены в [3] (верхние) и [15] (нижние). Используя оценку $A(1) \leqslant 0.8$, полученную в 1972 г. П. ван Биком [20], Михель [29] показал, что $C_{\mathrm{BE}}(1) \leqslant A(1) \leqslant 0.8$. Не будучи знакомыми с этой работой Михеля, авторы статьи [22], применив уточненное неравенство сглаживания Эссеена, получили оценку $C_{\mathrm{BE}}(1) \leqslant 1.99$. Из метода доказательства, использованного Михелем для $\delta=1$ (и обобщенного позже на случай произвольного $0<\delta \leqslant 1$ в книге [5]), вытекает, что если для абсолютной константы $A(\delta)$ в классическом неравенстве Берри-Эссеена с иентральныл ляпуновским отношением (2) справедлива оценка $A(\delta) \leqslant M(\delta)$, то та же оценка справедлива и для константы $C_{\mathrm{BE}}(\delta)$ в аналоге неравенства Берри-Эссеена 
с нецентральныл ляпуновским отношением (1) для пуассоновских случайных сумм: $C_{\mathrm{BE}}(\delta) \leqslant M(\delta)$. На это обстоятельство также обратили внимание в 1997 г. В.Ю.Королев и С.Я.Шоргин [28], которые независимо от Михеля [29] получили тот же результат, но с другой, лучшей на тот момент времени оценкой $M(1)=0.7655$, доказанной в 1982 г. И. С.Шигановым [19]. Поскольку наилучшая на сегодняшний день оценка абсолютной постоянной в классическом неравенстве БерриЭссеена имеет вид $A(1)<0.4748$ (см. [31]), можно заключить, следуя логике работ [29], [28], что неравенство (1) справедливо с $C_{\mathrm{BE}}(1)=0.4748$.

И лишь недавно, в 2009 г., В. Ю. Королеву и И. Г. Шевцовой [6] удалось показать, что на самом деле привязка оценки константы $C_{\mathrm{BE}}(1)$ в аналоге неравенства Берри-Эссеена для пуассоновских сумм (1) к оценке абсолютной постоянной $A(1)$ в классическом неравенстве БерриЭссеена (2) менее жесткая. А именно, в [6] было показано, что (1) справедливо с $C_{\mathrm{BE}}(1)=0.345$, и тем самым впервые было продемонстрировано, что абсолютная постоянная в аналоге неравенства Берри-Эссеена для пуассоновских случайных сумм (1) строго меньше минимально возможного значения $(\sqrt{10}+3) /(6 \sqrt{2 \pi})$ (см. [25]) абсолютной константы $A(1)$ в классическом неравенстве Берри-Эссеена (2). Позже те же авторы уточнили свою оценку до $C_{\mathrm{BE}}(1) \leqslant 0.3041$ (см. [27]). Аналогичный результат для $0<\delta \leqslant 1$ был получен в [10] (см. второй столбец таблицы 1). А для наилучшего возможного значения $C(\delta)$ при $\delta=0$

$$
C_{\mathrm{BE}}(0)=\sup _{\lambda>0, F \in \mathscr{F}_{2}} \Delta_{\lambda}(F)
$$

верхнюю оценку несложно получить из леммы 12.3 монографии Р. Н. Бхаттачария и Р. Ранга Рао [2]:

$$
C_{\mathrm{BE}}(0) \leqslant \sup _{F \in \mathscr{F}_{2}} \sup _{x}|F(x)-\Phi(x)| \leqslant \sup _{b>0}\left(\frac{1}{1+b^{2}}-\Phi(-b)\right)<0.54094 .
$$

Установленный в работе [6] факт обострил интерес к нижним оценкам постоянной $C_{\mathrm{BE}}(1)$ в $(1)$. Несмотря на то что нижняя оценка абсолютной константы $A(1)$ в классическом неравенстве Берри-Эссеена (2) известна еще с 1956 г. [25], нижних оценок для $C_{\mathrm{BE}}(1)$ и тем более для $C_{\mathrm{BE}}(\delta)$ при $0<\delta<1$ в (1) до недавнего времени известно не было. Указанный пробел был восполнен в [10] с помощью определения по аналогии с терминологией, использованной в работе [15], верхней асимптотически правильной константь:

$$
\bar{C}_{\mathrm{A \Pi}}\left(\mathscr{F}_{2+\delta}\right)=\limsup _{\lambda \rightarrow \infty} \sup _{F \in \mathscr{F}_{2+\delta}} \frac{\Delta_{\lambda}(F)}{\ell_{\lambda}(F)}, \quad 0 \leqslant \delta \leqslant 1,
$$


Таблица 1. Двусторонние оценки константы $C_{\mathrm{BE}}(\delta)$ в неравенстве БерриЭссеена и верхних асимптотически правильных констант $\bar{C}_{\mathrm{A \Pi}}\left(\mathscr{F}_{2+\delta}\right)$, $\bar{C}_{\mathrm{A \Pi}}\left(\mathscr{F}_{2+\delta, \mathrm{s}}\right)$, определенных в $(4)$, для некоторых $\delta$. По определению, $\bar{C}_{\mathrm{A \Pi}}\left(\mathscr{F}_{2+\delta, \mathrm{s}}\right) \leqslant \bar{C}_{\mathrm{A \Pi}}\left(\mathscr{F}_{2+\delta}\right) \leqslant C_{\mathrm{BE}}(\delta)$ для всех $0 \leqslant \delta \leqslant 1$.

\begin{tabular}{|c|c|c|c|}
\hline$\delta$ & $C_{\mathrm{BE}}(\delta) \leqslant$ & $\bar{C}_{\mathrm{A \Pi}}\left(\mathscr{F}_{2+\delta}\right) \geqslant$ & $\bar{C}_{\mathrm{A \Pi}}\left(\mathscr{F}_{2+\delta, s}\right) \geqslant$ \\
\hline 1.0 & 0.3041 & 0.2659 & 0.2344 \\
0.9 & 0.3089 & 0.2698 & 0.2383 \\
0.8 & 0.3187 & 0.2819 & 0.2446 \\
0.7 & 0.3334 & 0.2961 & 0.2534 \\
0.6 & 0.3538 & 0.3128 & 0.2651 \\
0.5 & 0.3775 & 0.3328 & 0.2803 \\
0.4 & 0.4080 & 0.3568 & 0.3000 \\
0.3 & 0.4450 & 0.3862 & 0.3257 \\
0.2 & 0.4901 & 0.4232 & 0.3603 \\
0.1 & 0.5451 & 0.4714 & 0.4097 \\
\hline
\end{tabular}

которая, очевидно, является минорантой для $C_{\mathrm{BE}}(\delta)$. Введем также верxнюю асимптотически правильную константу для симметричных распределений $\bar{C}_{\mathrm{A \Pi}}\left(\mathscr{F}_{2+\delta, \mathrm{s}}\right)$, которая определяется аналогично $\bar{C}_{\mathrm{A \Pi}}\left(\mathscr{F}_{2+\delta}\right)$ с заменой $\mathscr{F}_{2+\delta}$ на $\mathscr{F}_{2+\delta, \mathrm{s}}$. В работах [30], [10] найдены нижние оценки $\bar{C}_{\text {АП }}\left(\mathscr{F}_{2+\delta}\right)$ для $0<\delta \leqslant 1$, но, поскольку соответствующие экстремальные распределения симметричны, те же оценки остаются верными и для $\bar{C}_{\text {АП }}\left(\mathscr{F}_{2+\delta, \mathrm{s}}\right)$. Таким образом, из результатов работ [30], [10] (для $0<\delta \leqslant 1$ ) и нижеследующей теоремы 7 (для $\delta=0$ ) вытекает, что при Bcex $0 \leqslant \delta \leqslant 1$

$$
C_{\mathrm{BE}}(\delta) \geqslant \bar{C}_{\mathrm{A} \Pi}\left(\mathscr{F}_{2+\delta}\right) \geqslant \bar{C}_{\mathrm{A \Pi}}\left(\mathscr{F}_{2+\delta, \mathrm{s}}\right) \geqslant \frac{1}{2} \sup _{\gamma>0} \gamma^{\delta / 2} e^{-\gamma} I_{0}(\gamma)
$$

где $I_{0}(\gamma)=\sum_{k=0}^{\infty}\left(\gamma^{k} /\left(2^{k} k !\right)\right)^{2}$ и $\gamma^{\delta / 2} \equiv 1$ при $\delta=0$. В частности,

$\bar{C}_{\mathrm{A \Pi}}\left(\mathscr{F}_{3, \mathrm{~s}}\right) \geqslant \frac{1}{2} \sup _{\gamma>0} \sqrt{\gamma} e^{-\gamma} I_{0}(\gamma)>0.2344, \quad \bar{C}_{\mathrm{A \Pi}}\left(\mathscr{F}_{2, \mathrm{~s}}\right) \geqslant \frac{1}{2} \sup _{\gamma>0} e^{-\gamma} I_{0}(\gamma)=\frac{1}{2}$.

Значения минорант $\bar{C}_{\text {Ап }}\left(\mathscr{F}_{2+\delta, \mathrm{s}}\right)$ при других $0<\delta<1$ приведены в таблице 1 в четвертом столбце. Однако нижние оценки для $\bar{C}_{\mathrm{A \Pi}}\left(\mathscr{F}_{2+\delta}\right)$ и, следовательно, для $C_{\mathrm{BE}}(\delta)$, представленные здесь в терминах $\bar{C}_{\text {Ап }}\left(\mathscr{F}_{2+\delta, \mathrm{s}}\right)$, можно уточнить. А именно, в данной работе (см. теорему 5) будет показано, что при всех $0 \leqslant \delta \leqslant 1$

$$
C_{\mathrm{BE}}(\delta) \geqslant \bar{C}_{\mathrm{A \Pi}}\left(\mathscr{F}_{2+\delta}\right) \geqslant \sup _{\gamma>0, m \in \mathbf{N}_{0}} \gamma^{\delta / 2}\left(e^{-\gamma} \sum_{k=0}^{m} \frac{\gamma^{k}}{k !}-\Phi\left(\frac{m-\gamma}{\sqrt{\gamma}}\right)\right)
$$


где $\mathbf{N}_{0}$ - множество целых неотрицательных чисел (определение величины $C_{\mathrm{BE}}(0)$ см. в $\left.(3)\right)$. В частности,

$$
\begin{gathered}
C_{\mathrm{BE}}(1) \geqslant \bar{C}_{\mathrm{A} \Pi}\left(\mathscr{F}_{3}\right) \geqslant \frac{2}{3 \sqrt{2 \pi}}>0.2659, \\
C_{\mathrm{BE}}(0) \geqslant \bar{C}_{\mathrm{A} \Pi}\left(\mathscr{F}_{2}\right) \geqslant \sup _{\gamma>0}\left(e^{-\gamma}-\Phi(-\sqrt{\gamma})\right)=\sup _{b>0}\left(e^{-b^{2}}-\Phi(-b)\right)>0.54006,
\end{gathered}
$$

при других $0<\delta<1$ значения минорант $\bar{C}_{\text {АП }}\left(\mathscr{F}_{2+\delta}\right)$ указаны в таблице 1 в третьем столбце. Таким образом, для $\delta=0$ зазор между верхней 0.54094 и нижней 0.54006 оценками константы $C_{\mathrm{BE}}(0)$ довольно мал и не превосходит 0.0009. По всей видимости, верхнюю оценку константы $C_{\mathrm{BE}}(0)$ можно уточнить, так как использованная для этих целей в данной работе верхняя граница величины $\sup _{x}|F(x)-\Phi(x)|$ достигается на некотором двухточечном распределении $F \in \mathscr{F}_{2}$, которым обобщенное пуассоновское в силу своей безграничной делимости в принципе быть не может.

Заметим, что нижние оценки для $C_{\mathrm{BE}}(\delta), 0<\delta \leqslant 1$, установленные в работах [30], [10] и в нижеследующей теореме 5 в терминах верxних асимптотически правильных констант $\bar{C}_{\text {АП }}\left(\mathscr{F}_{2+\delta}\right), \bar{C}_{\text {АП }}\left(\mathscr{F}_{2+\delta, \mathrm{s}}\right)$, перестают, вообще говоря, быть справедливыми, если в (1) ограничиться классом функций распределения $F \in \mathscr{F}_{2+\delta}$, для которых ляпуновская дробь $\ell_{\lambda}(F)$ принимает малые или бесконечно малые значения. Чтобы устранить этот недостаток, по аналогии с предложенным в 1953 г. А. Н. Колмогоровым [4] подходом к вычислению наилучшего возможного значения константы в неравенстве Берри-Эссеена введем асимптотически правильнье константы в классах функций распределения $\mathscr{F}$, в качестве которых будут выступать $\mathscr{F}_{2+\delta}, \mathscr{F}_{2+\delta, \mathrm{s}}$ с $0<\delta \leqslant 1$ :

$$
C_{\mathrm{A} \Pi}(\mathscr{F})=\limsup _{\ell \rightarrow 0} \sup _{\lambda>0, F \in \mathscr{F}: \ell_{\lambda}(F)=\ell} \frac{\Delta_{\lambda}(F)}{\ell},
$$

а также - по аналогии с терминологией, использованной в работе [15], — нижние асимптотически правильные константы:

$$
\underline{C_{\mathrm{A \Pi}}}(\mathscr{F})=\limsup _{\ell \rightarrow 0} \limsup _{\lambda \rightarrow \infty} \sup _{F \in \mathscr{F}: \ell_{\lambda}(F)=\ell} \frac{\Delta_{\lambda}(F)}{\ell},
$$

обеспечивающие справедливость асимптотических оценок

$$
\begin{aligned}
& \Delta_{\lambda} \leqslant C_{\mathrm{A} \Pi}(\mathscr{F}) \ell_{\lambda}+o\left(\ell_{\lambda}\right), \lambda \rightarrow \infty, \\
& \Delta_{\lambda} \leqslant \underline{C_{\mathrm{A} \Pi}(\mathscr{F}) \ell_{\lambda}+K \lambda^{-\delta / 2}+o\left(\ell_{\lambda}\right),} \quad \lambda \rightarrow \infty,
\end{aligned}
$$

для некоторого $K \in \mathbf{R}$, не зависящего от $\lambda$ и $\ell_{\lambda}$, и всех $F \in \mathscr{F}$ таких, что $\ell_{\lambda}(F) \rightarrow 0$ при $\lambda \rightarrow \infty$. Эти асимптотические оценки, очевидно, 
являются наилучшими из возможных в отношении множителей при $\ell_{\lambda}$. Легко видеть, что определенные выше величины связаны неравенствами

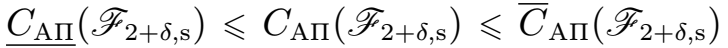

$$
\begin{aligned}
& \overline{\bar{\Lambda}} \quad \overline{\bar{\Lambda}} \quad \overline{\bar{\wedge}} \\
& \underline{C}_{\mathrm{A \Pi}}\left(\mathscr{F}_{2+\delta}\right) \leqslant C_{\mathrm{A} \Pi}\left(\mathscr{F}_{2+\delta}\right) \leqslant \bar{C}_{\mathrm{A \Pi}}\left(\mathscr{F}_{2+\delta}\right) \leqslant C_{\mathrm{BE}}(\delta), \quad 0<\delta \leqslant 1 .
\end{aligned}
$$

В работах [30], [9] показано, что

$$
\begin{array}{ll}
\underline{C}_{\mathrm{A} \Pi}\left(\mathscr{F}_{2+\delta, \mathrm{s}}\right) \\
\quad \geqslant \begin{cases}(2 \sqrt{2 \pi})^{-1}=0.1994 \ldots, & \delta=1, \\
\frac{\sqrt{\pi}}{2^{(5+\delta) / 2} \Gamma((3+\delta) / 2)} \sup _{s>0} \frac{\left(1+s^{2} / 2\right)^{-1}+s^{2} / 4-1}{s^{2+\delta}}, & 0<\delta<1\end{cases}
\end{array}
$$

(в формулировках соответствующих теорем в работах [30], [9] объявлены нижние оценки для $C_{\mathrm{A \Pi}}\left(\mathscr{F}_{2+\delta}\right)$, но фактически по ходу доказательства получены нижние оценки для $\left.C_{\mathrm{A \Pi}}\left(\mathscr{F}_{2+\delta, \mathrm{s}}\right)\right)$. Значения минорант в $(9)$ при некоторых $0<\delta<1$ приведены в таблице 2 .

В данной работе получены верхние оценки асимптотически правильных констант $C_{\mathrm{A \Pi}}\left(\mathscr{F}_{2+\delta}\right)$ (теорема 3$)$ и $C_{\mathrm{A} \Pi}\left(\mathscr{F}_{2+\delta, \mathrm{s}}\right)$ (теорема 4$) . \mathrm{B}$ частности, для $\delta=1$ в следствии 3 впервые найдено точное значение асимптотически правильной константы $C_{\text {Ап }}\left(\mathscr{F}_{3}\right)$ в аналоге неравенства Берри-Эссеена для пуассоновских случайных сумм и показано, что

$$
C_{\mathrm{A} \Pi}\left(\mathscr{F}_{3}\right)=\underline{C_{\mathrm{A} \Pi}}\left(\mathscr{F}_{3}\right)=\frac{2}{3 \sqrt{2 \pi}}=0.2659 \ldots
$$

Из этих соотношений вытекает, что асимптотическая оценка (8) не может улучшить при $\delta=1$ оценку (7), т.е. ни при каком выборе $K \in \mathbf{R}$ значение коэффициента при ляпуновской дроби в (8) не может быть сделано меньше, чем значение коэффициента при ляпуновской дроби в (7). Этот результат необычен тем, что для классической схемы суммирования детерминированного числа $n$ независимых одинаково распределенных случайных величин оценки, аналогичные (7) и (8) (в которых вместо $\lambda$ фигурирует $n$ ), различаются. Более того, в следствии 1 будет получена асимптотически правильная оценка

$$
\Delta_{\lambda} \leqslant \frac{2 \ell_{\lambda}}{3 \sqrt{2 \pi}}+0.4679 \cdot \ell_{\lambda}^{2}, \quad \lambda>0, \quad F \in \mathscr{F}_{3},
$$

неулучшаемая в отношении множителя $2 /(3 \sqrt{2 \pi})$ при $\ell_{\lambda}$, и тем самым конкретизирован вид $o\left(\ell_{\lambda}\right)$ в $(7)$. Эта асимптотическая оценка уточняет неравенство (1) с наилучшей известной на данный момент оценкой 
$C_{\mathrm{BE}}(1) \leqslant 0.3041$ при всех $\ell_{\lambda} \leqslant 0.08$. В следствии 2 показано, что для симметричного случая полученную в следствии 1 оценку можно уточнить до

$$
\begin{aligned}
\Delta_{\lambda} & \leqslant \frac{1+2 \varkappa_{1}}{2 \sqrt{2 \pi}} \ell_{\lambda}+0.3442 \cdot \ell_{\lambda}^{2} \\
& <0.2391 \cdot \ell_{\lambda}+0.3442 \cdot \ell_{\lambda}^{2}, \quad \lambda>0, \quad F \in \mathscr{F}_{3, \mathrm{~s}},
\end{aligned}
$$

где $\varkappa_{1}=\sup _{x>0}\left(\cos x-1+x^{2} / 2\right) / x^{3}<0.0992$. Как вытекает из (9), значение коэффициента при $\ell_{\lambda}$ в этой оценке не может быть меньше, чем $(2 \sqrt{2 \pi})^{-1}=0.1994 \ldots$. В теоремах 1 и 2 получены аналогичные результаты для случая, когда соответственно $F \in \mathscr{F}_{2+\delta}$ и $F \in \mathscr{F}_{2+\delta, \mathrm{s}}$ с произвольным $0<\delta \leqslant 1$.

\section{2. Верхние оценки.}

Теорема 1. Для $0<\delta \leqslant 1$ обозначим $\theta_{0}(\delta)$ единственный корень уравнения

$$
\delta \theta^{2}+2 \theta \sin \theta+2(2+\delta)(\cos \theta-1)=0, \quad \pi<\theta<2 \pi .
$$

Пусть

$$
\begin{aligned}
\varkappa_{\delta}= & \sup _{x>0} \frac{\left|\cos x-1+x^{2} / 2\right|}{x^{2+\delta}}=\frac{\cos \theta_{0}(\delta)-1+\theta_{0}^{2}(\delta) / 2}{\theta_{0}^{2+\delta}(\delta)}=\frac{\theta_{0}(\delta)-\sin \theta_{0}(\delta)}{(2+\delta) \theta_{0}^{1+\delta}(\delta)}, \\
\gamma_{\delta}= & \sup _{x>0} \sqrt{\left(\frac{\cos x-1+x^{2} / 2}{x^{2+\delta}}\right)^{2}+\left(\frac{\sin x-x}{x^{2+\delta}}\right)^{2}}, \\
t_{1}(\delta)= & \theta_{0}(\delta) /(2 \pi), t_{2}=t_{2}(\delta)-\text { единственный корень уравнения } \\
& t^{2}\left(1-2 \varkappa_{\delta}(2 \pi t)^{\delta}\right)=t_{1}^{2}(\delta)\left(1-2 \varkappa_{\delta}\left(2 \pi t_{1}(\delta)\right)^{\delta}\right), \quad 0<t<t_{1}(\delta),
\end{aligned}
$$

$u$

$$
t_{3}(\delta)=\frac{1}{2 \pi}\left(\frac{2}{(2+\delta)^{2} \varkappa_{\delta}}\right)^{1 / \delta} \exp \left\{-\frac{4+\delta}{2(2+\delta)}\right\}
$$

а также

$$
\begin{aligned}
& J_{21}\left(T, t_{0}\right)=\frac{1.0253}{\pi} T^{2} \int_{t_{0} \wedge t_{2}(\delta)}^{t_{2}(\delta)} \exp \left\{-\frac{T^{2} t^{2}}{2}\left(1-2 \varkappa_{\delta}(2 \pi t)^{\delta}\right)\right\} \frac{d t}{t}, \\
& J_{22}\left(T, t_{0}\right)= \frac{1.0253}{\pi} T^{2} \exp \left\{-\frac{T^{2} t_{1}^{2}(\delta)}{2}\left(1-2 \varkappa_{\delta}\left(2 \pi t_{1}(\delta)\right)^{\delta}\right)\right\} \ln \frac{t_{1}(\delta)}{t_{0} \vee t_{2}(\delta)} \\
& J_{3}(T)=T^{2} \int_{0}^{1-t_{1}(\delta)} t \sqrt{1+\left(\frac{1}{\pi\left(t \vee 10^{-4}\right)}-\cot \pi\left(t \vee 10^{-4}\right)\right)^{2}} \\
& \times \exp \left\{-T^{2} \frac{1-\cos 2 \pi t}{4 \pi^{2}}\right\} d t
\end{aligned}
$$




$$
\begin{aligned}
J_{4}\left(T, t_{0}\right)=0 \vee & \frac{1}{t_{0}}\left(\frac{1}{\pi t_{0}}-1+t_{0}-\frac{\pi^{2} t_{0}^{2}}{18}\right) e^{-T^{2} t_{0}^{2} / 2}, \\
J\left(T, t_{0}\right)=0 \vee( & J_{21}\left(T, t_{0}\right)+J_{22}\left(T, t_{0}\right)+J_{3}(T) \\
& \left.\quad+J_{4}\left(T, t_{0}\right)-1+\frac{\pi^{5 / 2}}{18 \sqrt{2}} \cdot \frac{1}{T}\right), \quad T>0 .
\end{aligned}
$$

Тогда для любого $\ell>0$ при всех $\lambda>0$ u $F \in \mathscr{F}_{2+\delta}$ таких, что $\ell_{\lambda} \leqslant \ell$, справедлива оченка

$$
\Delta_{\lambda} \leqslant C(\delta) \ell_{\lambda}+\frac{\ell_{\lambda}^{1 / \delta}}{2 \sqrt{2 \pi}}+A_{\delta}\left(\ell \wedge \ell_{*}(\delta)\right) \cdot \ell_{\lambda}^{2}
$$

гдe

$$
\begin{gathered}
C(\delta)=\frac{\gamma_{\delta} \cdot 2^{\delta / 2}}{\pi} \Gamma\left(\frac{2+\delta}{2}\right), \\
A_{\delta}(\ell)=\ell^{(1-\delta) / \delta} \cdot 2^{(\delta-1) / 2} \pi^{-1} \gamma_{\delta} \Gamma\left(\frac{3+\delta}{2}\right)\left(1+\frac{(3+\delta) \ell^{2 / \delta}}{72}\right) \\
+\inf _{t_{3}(\delta) \leqslant t_{0}<t_{1}(\delta)}\left(1.0253 \frac{2^{3 / 2+\delta} \varkappa_{\delta} \gamma_{\delta} \Gamma(5 / 2+\delta)}{\pi\left(1-2 \varkappa_{\delta}\left(2 \pi t_{0}\right)^{\delta}\right)^{5 / 2+\delta}}\right. \\
\left.+\frac{\ell^{2(1-\delta) / \delta}}{4 \pi^{2}} \sup _{0<\varepsilon \leqslant \ell} J\left(\frac{2 \pi}{\varepsilon^{1 / \delta}}, t_{0}\right)\right),
\end{gathered}
$$

$\Gamma(\cdot)$ - эйлерова гамма- функиия; $\ell_{*}(\delta)$ - единственный корень уравнения

$$
\begin{gathered}
A_{\delta}(\ell) \ell^{2}=\kappa-C(\delta) \ell-\frac{\ell^{1 / \delta}}{2 \sqrt{2 \pi}}, \quad 0<\ell<(2 \sqrt{2 \pi} \kappa)^{\delta}, \\
\kappa \equiv \sup _{F \in \mathscr{F}_{2}} \sup _{x}|F(x)-\Phi(x)|=\sup _{b>0}\left(\frac{1}{1+b^{2}}-\Phi(-b)\right)=0.54093 \ldots
\end{gathered}
$$

Значения величины $C(\delta)$ при некоторых $0<\delta \leqslant 1$ приведены в таблице 2 во втором и шестом столбцах, значения $\ell_{*}(\delta), A_{\delta}(\ell)$, округленные в бо́льшую сторону с точностью до четвертого знака, - в таблице 3 , а значения величин $\gamma_{\delta}, \varkappa_{\delta}, t_{1}(\delta), t_{2}(\delta), t_{3}(\delta)$ с точностью до четвертого знака — в таблице 4.

Теорема 2. Для любых $0<\delta \leqslant 1$ u $\ell>0$ при всех $\lambda>0 u F \in$ $\mathscr{F}_{2+\delta, \mathrm{s}}$ таких, что $\ell_{\lambda} \leqslant \ell$, справедлива оченка

$$
\Delta_{\lambda} \leqslant C_{\mathrm{s}}(\delta) \ell_{\lambda}+\frac{\ell_{\lambda}^{1 / \delta}}{2 \sqrt{2 \pi}}+A_{\delta}^{\mathrm{s}}\left(\ell \wedge \ell_{*}^{\mathrm{s}}(\delta)\right) \cdot \ell_{\lambda}^{2}
$$

где

$$
C_{\mathrm{s}}(\delta)=\frac{\varkappa_{\delta} \cdot 2^{\delta / 2}}{\pi} \Gamma\left(\frac{2+\delta}{2}\right)
$$




$$
\begin{aligned}
& A_{\delta}^{\mathrm{s}}(\ell)=\ell^{(1-\delta) / \delta} \cdot 2^{(\delta-1) / 2} \pi^{-1} \varkappa_{\delta} \Gamma\left(\frac{3+\delta}{2}\right)\left(1+\frac{(3+\delta) \ell^{2 / \delta}}{72}\right) \\
&+\inf _{t_{3}(\delta) \leqslant t_{0}<t_{1}(\delta)}( 1.0253 \frac{2^{3 / 2+\delta} \varkappa_{\delta}^{2} \Gamma(5 / 2+\delta)}{\pi\left(1-2 \varkappa_{\delta}\left(2 \pi t_{0}\right)^{\delta}\right)^{5 / 2+\delta}} \\
&\left.+\frac{\ell^{2(1-\delta) / \delta}}{4 \pi^{2}} \sup _{0<\varepsilon \leqslant \ell} J\left(\frac{2 \pi}{\varepsilon^{1 / \delta}}, t_{0}\right)\right),
\end{aligned}
$$

$\ell_{*}^{\mathrm{s}}(\delta)-$ единственный корень уравнения

$$
A_{\delta}^{\mathrm{s}}(\ell) \ell^{2}=\kappa-C_{\mathrm{s}}(\delta) \ell-\frac{\ell^{1 / \delta}}{2 \sqrt{2 \pi}}, \quad 0<\ell<(2 \sqrt{2 \pi} \kappa)^{\delta},
$$

$\kappa=0.5409 \ldots$, величинь $\varkappa_{\delta}, t_{1}(\delta), t_{3}(\delta), J\left(T, t_{0}\right), T>0$, определены в формулировке теоремь 1.

Таблица 2. Значения величин $C(\delta), C_{\mathrm{S}}(\delta)$ из теорем 1 и 2 , мажорирующих асимптотически правильные константы $C_{\mathrm{A \Pi}}\left(\mathscr{F}_{2+\delta}\right)$ и $C_{\mathrm{A \Pi}}\left(\mathscr{F}_{2+\delta, \mathrm{s}}\right)(\mathrm{cм.}$ теоремы 3 и 4), рядом - соответствующие значения миноранты нижней асимптотически правильной константы $C_{\mathrm{A \Pi}}\left(\mathscr{F}_{2+\delta, \mathrm{s}}\right)$ (см. $\left.(9)\right)$ при некоторых $0<\delta<1$. По определению, $\underline{C}_{\mathrm{A \Pi}}\left(\overline{\mathscr{F}}_{2+\delta, \mathrm{s}}\right) \leqslant C_{\mathrm{A} \Pi}\left(\mathscr{F}_{2+\delta, \mathrm{s}}\right) \leqslant C_{\mathrm{s}}(\delta)$, $\underline{C}_{\mathrm{A} \Pi}\left(\mathscr{F}_{2+\delta, \mathrm{s}}\right) \leqslant C_{\mathrm{A} \Pi}\left(\mathscr{F}_{2+\delta}\right) \leqslant C(\delta)$ для всех $0<\delta \leqslant 1$.

\begin{tabular}{||c|c|c|c||c|c|c|c||}
\hline$\delta=$ & $C(\delta) \leqslant$ & $C_{\mathrm{s}}(\delta) \leqslant$ & $C_{\mathrm{A} \Pi}\left(\mathscr{F}_{2+\delta, \mathrm{s}}\right) \geqslant$ & $\delta=$ & $C(\delta) \leqslant$ & $C_{\mathrm{s}}(\delta) \leqslant$ & $C_{\mathrm{A} \Pi}\left(\mathscr{F}_{2+\delta, \mathrm{s}}\right) \geqslant$ \\
\hline $0+$ & 0.1693 & 0.1592 & 0.0883 & 0.50 & 0.0854 & 0.0724 & 0.0308 \\
0.05 & 0.1561 & 0.1458 & 0.0747 & 0.55 & 0.0810 & 0.0676 & 0.0286 \\
0.10 & 0.1444 & 0.1338 & 0.0655 & 0.60 & 0.0772 & 0.0632 & 0.0266 \\
0.15 & 0.1339 & 0.1231 & 0.0583 & 0.65 & 0.0738 & 0.0593 & 0.0248 \\
0.20 & 0.1245 & 0.1135 & 0.0524 & 0.70 & 0.0709 & 0.0556 & 0.0232 \\
0.25 & 0.1161 & 0.1048 & 0.0474 & 0.75 & 0.0685 & 0.0523 & 0.0217 \\
0.30 & 0.1085 & 0.0969 & 0.0431 & 0.80 & 0.0665 & 0.0493 & 0.0204 \\
0.35 & 0.1017 & 0.0899 & 0.0394 & 0.85 & 0.0650 & 0.0466 & 0.0192 \\
0.40 & 0.0956 & 0.0835 & 0.0361 & 0.90 & 0.0642 & 0.0440 & 0.0182 \\
0.45 & 0.0902 & 0.0777 & 0.0333 & 0.95 & 0.0642 & 0.0417 & 0.0172 \\
& & & & $1-$ & 0.0665 & 0.0396 & 0.0163 \\
\hline
\end{tabular}

Значения величины $C_{\mathrm{s}}(\delta)$ при некоторых $0<\delta \leqslant 1$ приведены в таблице 2 в третьем и седьмом столбцах, а значения $\ell_{*}^{\mathrm{s}}(\delta), A_{\delta}^{\mathrm{s}}(\ell)$, округленные в бо́льшую сторону с точностью до четвертого знака, - в таблице 3.

Поскольку $C(1)=(6 \sqrt{2 \pi})^{-1}, C_{\mathrm{s}}(1)=\varkappa_{1} / \sqrt{2 \pi}$, из теорем 1 и 2 при $\delta=1$ получаем два следствия.

Следствие 1. Для любых $\ell>0 u \lambda>0$ при всех $F \in \mathscr{F}_{3}$ таких, что $\ell_{\lambda} \leqslant \ell$, справедливы оченки

$$
\Delta_{\lambda} \leqslant \frac{2 \ell_{\lambda}}{3 \sqrt{2 \pi}}+A_{1}(\ell \wedge 0.8280) \cdot \ell_{\lambda}^{2}<0.2660 \cdot \ell_{\lambda}+0.4679 \cdot \ell_{\lambda}^{2}
$$


c $A_{1}(\ell)$, определенной в формулировке теоремь 1. B частности, $A_{1}(0.1) \leqslant 0.2684$ и при $\ell_{\lambda} \leqslant 0.1$

$$
\Delta_{\lambda} \leqslant \frac{2 \ell_{\lambda}}{3 \sqrt{2 \pi}}+0.2684 \cdot \ell_{\lambda}^{2}< \begin{cases}0.2929 \cdot \ell_{\lambda}, & \ell_{\lambda} \leqslant 0.1, \\ 0.2687 \cdot \ell_{\lambda}, & \ell_{\lambda} \leqslant 10^{-2} \\ 0.2663 \cdot \ell_{\lambda}, & \ell_{\lambda} \leqslant 10^{-3} \\ 0.2660 \cdot \ell_{\lambda}, & \ell_{\lambda} \leqslant 10^{-4}\end{cases}
$$

3 а м е ч а н и е 1 . Как будет следовать из теоремы 6 , значение коэффициента $2 /(3 \sqrt{2 \pi})$ при ляпуновской дроби $\ell_{\lambda}$ в $(12)$ не может быть уменьшено.

Таблица 3. Значения величин $\ell_{*}(\delta)$ и $A_{\delta}(\ell)$ из теоремы 1 для $\ell=\ell_{*}(\delta), 0.5 \wedge$ $\ell_{*}(\delta), 0.1 \wedge \ell_{*}(\delta)$ и $\ell \rightarrow 0+$ при некоторых $0<\delta \leqslant 1$. В третьем столбце указаны оптимальные значения $t_{0}$, доставляющие инфимум в $A_{\delta}\left(\ell_{*}(\delta)\right)$, при других $\ell$ оптимальные значения $t_{0}$ совпадают с $t_{3}(\delta)$ (см. таблицу 4$)$.

\begin{tabular}{|c|c|c|c|c|c|c|}
\hline$\delta=$ & $\ell_{*}(\delta) \leqslant$ & $t_{0}=$ & $A_{\delta}\left(\ell_{*}(\delta)\right) \leqslant$ & $A_{\delta}(0.5) \leqslant$ & $A_{\delta}(0.1) \leqslant$ & $A_{\delta}(0+) \leqslant$ \\
\hline 0.05 & 0.0661 & 0.1370 & 121.7947 & 121.7947 & 121.7947 & 121.7947 \\
0.10 & 0.1477 & 0.1386 & 23.8244 & 23.8244 & 23.8244 & 23.8244 \\
0.15 & 0.2334 & 0.1401 & 9.3589 & 9.3589 & 9.3589 & 9.3589 \\
0.20 & 0.3205 & 0.1416 & 4.8734 & 4.8734 & 4.8726 & 4.8726 \\
0.25 & 0.4062 & 0.1431 & 2.9613 & 2.9613 & 2.9561 & 2.9561 \\
0.30 & 0.4863 & 0.1444 & 1.9884 & 1.9884 & 1.9750 & 1.9746 \\
0.35 & 0.5581 & 0.1457 & 1.4339 & 1.4291 & 1.4106 & 1.4096 \\
0.40 & 0.6170 & 0.1469 & 1.1095 & 1.0805 & 1.0588 & 1.0566 \\
0.45 & 0.6577 & 0.1480 & 0.9320 & 0.8508 & 0.8263 & 0.8225 \\
0.50 & 0.6867 & 0.1490 & 0.8237 & 0.6935 & 0.6660 & 0.6599 \\
0.55 & 0.7094 & 0.1500 & 0.7485 & 0.5833 & 0.5519 & 0.5429 \\
0.60 & 0.7283 & 0.1513 & 0.6924 & 0.5051 & 0.4686 & 0.4564 \\
0.65 & 0.7457 & 0.1621 & 0.6456 & 0.4497 & 0.4068 & 0.3909 \\
0.70 & 0.7628 & 0.1717 & 0.6040 & 0.4110 & 0.3605 & 0.3406 \\
0.75 & 0.7794 & 0.1801 & 0.5673 & 0.3847 & 0.3256 & 0.3015 \\
0.80 & 0.7950 & 0.1874 & 0.5356 & 0.3680 & 0.2997 & 0.2710 \\
0.85 & 0.8091 & 0.1937 & 0.5087 & 0.3565 & 0.2811 & 0.2474 \\
0.90 & 0.8209 & 0.1988 & 0.4870 & 0.3492 & 0.2687 & 0.2297 \\
0.95 & 0.8291 & 0.2026 & 0.4714 & 0.3469 & 0.2628 & 0.2176 \\
1.00 & 0.8280 & 0.2044 & 0.4679 & 0.3559 & 0.2684 & 0.2680 \\
\hline
\end{tabular}

Следствие 2. Для любых $\ell>0 u \lambda>0$ при всех $F \in \mathscr{F}_{3, \mathrm{~s}}$ maких, что $\ell_{\lambda} \leqslant \ell$, справедливь оченки

$$
\Delta_{\lambda} \leqslant \frac{1+2 \varkappa_{1}}{2 \sqrt{2 \pi}} \ell_{\lambda}+A_{1}^{s}(\ell \wedge 0.9537) \cdot \ell_{\lambda}^{2}<0.2391 \cdot \ell_{\lambda}+0.3442 \cdot \ell_{\lambda}^{2}
$$


с $A_{1}^{\mathrm{s}}(\ell)$, определенной в формулировке теоремь 2. В частности, $A_{1}^{\mathrm{s}}(0.1) \leqslant 0.1599$ и при $\ell_{\lambda} \leqslant 0.1$

$$
\Delta_{\lambda} \leqslant \frac{1+2 \varkappa_{1}}{2 \sqrt{2 \pi}} \ell_{\lambda}+0.1599 \cdot \ell_{\lambda}^{2}< \begin{cases}0.2551 \cdot \ell_{\lambda}, & \ell_{\lambda} \leqslant 0.1, \\ 0.2407 \cdot \ell_{\lambda}, & \ell_{\lambda} \leqslant 0.01, \\ 0.2392 \cdot \ell_{\lambda}, & \ell_{\lambda} \leqslant 0.001, \\ 0.2391 \cdot \ell_{\lambda}, & \ell_{\lambda} \leqslant 0.0004 .\end{cases}
$$

Таблица 4. Значения $\gamma_{\delta}, \varkappa_{\delta}, t_{1}(\delta), t_{2}(\delta), t_{3}(\delta)$ с точностью до четвертого знака для некоторых $0<\delta \leqslant 1$.

\begin{tabular}{|c|c|c|c|c|c|}
\hline$\delta$ & $\gamma_{\delta}$ & $\varkappa_{\delta}$ & $t_{1}(\delta)$ & $t_{2}(\delta)$ & $t_{3}(\delta)$ \\
\hline 0.01 & 0.5225 & 0.4909 & 0.9950 & 0.0261 & 0.1356 \\
0.05 & 0.4885 & 0.4563 & 0.9761 & 0.0673 & 0.1370 \\
0.10 & 0.4498 & 0.4170 & 0.9539 & 0.1019 & 0.1386 \\
0.15 & 0.4149 & 0.3815 & 0.9331 & 0.1302 & 0.1401 \\
0.20 & 0.3833 & 0.3494 & 0.9132 & 0.1551 & 0.1416 \\
0.25 & 0.3548 & 0.3203 & 0.8941 & 0.1778 & 0.1431 \\
0.30 & 0.3290 & 0.2940 & 0.8756 & 0.1989 & 0.1444 \\
0.35 & 0.3058 & 0.2701 & 0.8576 & 0.2187 & 0.1457 \\
0.40 & 0.2847 & 0.2484 & 0.8399 & 0.2375 & 0.1469 \\
0.45 & 0.2657 & 0.2287 & 0.8226 & 0.2556 & 0.1480 \\
0.50 & 0.2486 & 0.2108 & 0.8054 & 0.2729 & 0.1490 \\
0.55 & 0.2331 & 0.1945 & 0.7884 & 0.2896 & 0.1500 \\
0.60 & 0.2193 & 0.1796 & 0.7716 & 0.3058 & 0.1509 \\
0.65 & 0.2070 & 0.1661 & 0.7548 & 0.3214 & 0.1517 \\
0.70 & 0.1960 & 0.1537 & 0.7380 & 0.3366 & 0.1524 \\
0.75 & 0.1865 & 0.1424 & 0.7212 & 0.3514 & 0.1530 \\
0.80 & 0.1783 & 0.1321 & 0.7044 & 0.3657 & 0.1536 \\
0.85 & 0.1715 & 0.1227 & 0.6875 & 0.3797 & 0.1540 \\
0.90 & 0.1665 & 0.1142 & 0.6705 & 0.3932 & 0.1544 \\
0.95 & 0.1637 & 0.1063 & 0.6533 & 0.4064 & 0.1547 \\
1.00 & 0.1666 & 0.0991 & 0.6359 & 0.4191 & 0.1550 \\
\hline
\end{tabular}

Устремляя $\ell$ к нулю, из теорем 1 и 2 можно получить верхние оценки асимптотически правильных констант

$$
\begin{aligned}
C_{\mathrm{A} \Pi}\left(\mathscr{F}_{2+\delta}\right)=\limsup _{\ell \rightarrow 0} \sup _{\lambda>0, F \in \mathscr{F}_{2+\delta}: \ell_{\lambda}=\ell} \frac{\Delta_{\lambda}(F)}{\ell}, & 0<\delta \leqslant 1, \\
C_{\mathrm{A} \Pi}\left(\mathscr{F}_{2+\delta, \mathrm{s}}\right)=\limsup _{\ell \rightarrow 0} \sup _{\lambda>0, F \in \mathscr{F}_{2+\delta, s}: \ell_{\lambda}=\ell} \frac{\Delta_{\lambda}(F)}{\ell}, & 0<\delta \leqslant 1 .
\end{aligned}
$$


Таблица 5. Значения величин $\ell_{*}^{\mathrm{s}}(\delta)$ и $A_{\delta}^{\mathrm{s}}(\ell)$ из теоремы 2 для $\ell=\ell_{*}^{\mathrm{s}}(\delta), 0.5 \wedge$ $\ell_{*}^{\mathrm{s}}(\delta), 0.1 \wedge \ell_{*}^{\mathrm{s}}(\delta)$ и $\ell \rightarrow 0+$ при некоторых $0<\delta \leqslant 1$. В третьем столбце указаны оптимальные значения $t_{0}$, доставляющие инфимум в $A_{\delta}^{s}\left(\ell_{*}^{\mathrm{s}}(\delta)\right)$, при других $\ell$ оптимальные значения $t_{0}$ совпадают с $t_{3}(\delta)$ (см. таблицу 4$)$.

\begin{tabular}{|c|c|c|c|c|c|c|}
\hline$\delta$ & $\ell_{*}^{\mathrm{s}}(\delta)$ & $t_{0}=$ & $A_{\delta}^{\mathrm{s}}\left(\ell_{*}^{\mathrm{s}}(\delta)\right) \leqslant$ & $A_{\delta}^{\mathrm{s}}(0.5) \leqslant$ & $A_{\delta}^{\mathrm{s}}(0.1) \leqslant$ & $A_{\delta}^{\mathrm{s}}(0+) \leqslant$ \\
\hline 0.05 & 0.0684 & 0.1370 & 113.7650 & 113.7650 & 113.7650 & 113.7650 \\
0.10 & 0.1535 & 0.1386 & 22.0866 & 22.0866 & 22.0866 & 22.0866 \\
0.15 & 0.2437 & 0.1401 & 8.6060 & 8.6060 & 8.6060 & 8.6060 \\
0.20 & 0.3362 & 0.1416 & 4.4423 & 4.4423 & 4.4413 & 4.4413 \\
0.25 & 0.4279 & 0.1431 & 2.6743 & 2.6743 & 2.6689 & 2.6688 \\
0.30 & 0.5139 & 0.1444 & 1.7783 & 1.7775 & 1.7646 & 1.7643 \\
0.35 & 0.5907 & 0.1457 & 1.2716 & 1.2625 & 1.2462 & 1.2453 \\
0.40 & 0.6486 & 0.1469 & 0.9967 & 0.9428 & 0.9238 & 0.9220 \\
0.45 & 0.6870 & 0.1480 & 0.8499 & 0.7325 & 0.7113 & 0.7080 \\
0.50 & 0.7156 & 0.1490 & 0.7559 & 0.5886 & 0.5648 & 0.5596 \\
0.55 & 0.7392 & 0.1500 & 0.6879 & 0.4877 & 0.4603 & 0.4529 \\
0.60 & 0.7611 & 0.1616 & 0.6325 & 0.4160 & 0.3838 & 0.3738 \\
0.65 & 0.7838 & 0.1735 & 0.5820 & 0.3650 & 0.3264 & 0.3137 \\
0.70 & 0.8071 & 0.1842 & 0.5362 & 0.3289 & 0.2827 & 0.2671 \\
0.75 & 0.8310 & 0.1939 & 0.4949 & 0.3037 & 0.2488 & 0.2303 \\
0.80 & 0.8553 & 0.2025 & 0.4577 & 0.2845 & 0.2222 & 0.2009 \\
0.85 & 0.8799 & 0.2102 & 0.4243 & 0.2679 & 0.2012 & 0.1771 \\
0.90 & 0.9046 & 0.2171 & 0.3945 & 0.2534 & 0.1843 & 0.1575 \\
0.95 & 0.9292 & 0.2232 & 0.3679 & 0.2408 & 0.1708 & 0.1414 \\
1.00 & 0.9537 & 0.2286 & 0.3442 & 0.2299 & 0.1599 & 0.1595 \\
\hline & & & & & & \\
\hline
\end{tabular}

Теорема 3. Для асимптотически правильных констант $C_{\mathrm{A \Pi}}\left(\mathscr{F}_{2+\delta}\right)$ при всех $0<\delta \leqslant 1$ справедливы оченки

$$
C_{\mathrm{A} \Pi}\left(\mathscr{F}_{3}\right) \leqslant \frac{2}{3 \sqrt{2 \pi}}<0.2660, \quad C_{\mathrm{A} \Pi}\left(\mathscr{F}_{2+\delta}\right) \leqslant C(\delta), \quad 0<\delta<1,
$$

с $C(\delta)$, определенной в формулировке теоремь 1. В частности,

$$
\lim _{\delta \rightarrow 1-} C_{\mathrm{A} \Pi}\left(\mathscr{F}_{2+\delta}\right) \leqslant \frac{1}{6 \sqrt{2 \pi}}<0.0665, \quad \lim _{\delta \rightarrow 0+} C_{\mathrm{A} \Pi}\left(\mathscr{F}_{2+\delta}\right) \leqslant \frac{\gamma_{0}}{\pi}<0.1693 .
$$

Значения $C(\delta)$ для других $0<\delta<1$ приведень в табличе 2 .

Теорема 4. Для асимптотически правильных констант $C_{\mathrm{A \Pi}}\left(\mathscr{F}_{2+\delta, \mathrm{s}}\right)$ при всех $0<\delta \leqslant 1$ справедливы оченки

$$
C_{\mathrm{A} \Pi}\left(\mathscr{F}_{3, \mathrm{~s}}\right) \leqslant \frac{1+2 \varkappa_{1}}{2 \sqrt{2 \pi}}<0.2391, \quad C_{\mathrm{A} \Pi}\left(\mathscr{F}_{2+\delta, \mathrm{s}}\right) \leqslant C_{\mathrm{s}}(\delta), \quad 0<\delta<1,
$$


c $C_{\mathrm{s}}(\delta)$, определенной в формулировке теоремь 2. В частности,

$\lim _{\delta \rightarrow 1-} C_{\mathrm{A} \Pi}\left(\mathscr{F}_{2+\delta, \mathrm{s}}\right) \leqslant \frac{\varkappa_{1}}{\sqrt{2 \pi}}<0.0396, \quad \lim _{\delta \rightarrow 0+} C_{\mathrm{A} \Pi}\left(\mathscr{F}_{2+\delta, \mathrm{s}}\right) \leqslant \frac{\varkappa_{0}}{\pi}=\frac{1}{2 \pi}<0.1592$.

Значения $C_{\mathrm{s}}(\delta)$ для других $0<\delta<1$ приведень в табличе 2.

В следующем пункте будет показано, что $C_{\text {АП }}\left(\mathscr{F}_{3}\right)=2(3 \sqrt{2 \pi})^{-1}=$ $0.2659 \ldots$ (см. следствие 3 ), т.е. теорема 3 фактически устанавливает точное значение асимптотически правильной константы $C_{\mathrm{Aп}}\left(\mathscr{F}_{3}\right)$.

Доказательство теорем 1 и 2 основано на свойстве безграничной делимости обобщенного пуассоновского распределения и соответствующих оценках точности нормальной аппроксимации для сумм детерминированного числа независимых одинаково распределенных случайных величин. Последние даны в следующей лемме.

Пусть $Y, Y_{1}, Y_{2}, \ldots$ - независимые одинаково распределенные случайные величины, заданные на некотором вероятностном пространстве и имеющие общую функцию распределения $G \in \mathscr{F}_{2}$. Для $n=1,2, \ldots$ обозначим

$$
\begin{gathered}
\Delta_{n}^{*}=\Delta_{n}^{*}(Y)=\sup _{x \in \mathbf{R}}\left|G_{n}(x)-\Phi(x)\right|, \\
G_{n}(x)=\mathbf{P}\left(Y_{1}+\cdots+Y_{n}-n \mathbf{E} Y<x \sqrt{n \mathbf{D} Y}\right) \\
=G^{* n}(x \sqrt{n \mathbf{D} Y}+n \mathbf{E} Y), \quad x \in \mathbf{R},
\end{gathered}
$$

где $G^{* n}$ есть $n$-кратная свертка функции распределения $G$ с собой.

Лемма 1 (см. [32], [18]). Для любых $0<\delta \leqslant 1, \ell>0$ и произвольной бесконечно малой последовательности неотричательных чисел $\left\{\theta_{n}\right\}_{n \geqslant 1}$ справедливы соотношения

$$
\begin{aligned}
& \limsup _{n \rightarrow \infty} \sup _{Y \in \mathscr{F}_{2+\delta}:\left|L_{0}(Y) n^{-\delta / 2}-\ell\right| \leqslant \theta_{n}} \Delta_{n}^{*}(Y) \leqslant C(\delta) \ell+\frac{\ell^{1 / \delta}}{2 \sqrt{2 \pi}}+A_{\delta}\left(\ell \wedge \ell_{*}(\delta)\right) \cdot \ell^{2}, \\
& \limsup _{n \rightarrow \infty} \sup _{Y \in \mathscr{F}_{2+\delta, \mathrm{s}}:\left|L_{0}(Y) n^{-\delta / 2}-\ell\right| \leqslant \theta_{n}} \Delta_{n}^{*}(Y) \leqslant C_{\mathrm{s}}(\delta) \ell+\frac{\ell^{1 / \delta}}{2 \sqrt{2 \pi}}+A_{\delta}^{\mathrm{s}}\left(\ell \wedge \ell_{*}^{\mathrm{s}}(\delta)\right) \cdot \ell^{2},
\end{aligned}
$$

с $C(\delta), A_{\delta}(\ell), \ell_{*}(\delta)$, определенныли в формулировке теоремь $1, u C_{\mathrm{s}}(\delta)$, $A_{\delta}^{\mathrm{s}}(\ell), \ell_{*}^{\mathrm{s}}(\delta)$, определенньми в формулировке теоремь 2.

Свойство безграничной делимости с вытекающими из него оценками первых моментов сформулировано в следующей лемме (также cм. [13], [5]).

Лемма 2. Для любых $0<\delta \leqslant 1, F \in \mathscr{F}_{2+\delta}, \lambda>0 u n \geqslant 1$ существует вероятностное пространство и набор $n$ независимьх одинаково распределенных случайных величин $Y_{\nu, 1}, \ldots, Y_{\nu, n} \in \mathscr{F}_{2+\delta}, \nu=\lambda / n$, 
на нем таких, что

$$
\widetilde{S}_{\lambda} \stackrel{d}{=} \frac{1}{\sqrt{n}} \sum_{j=1}^{n} Y_{\nu, j}, \quad \mathbf{E} Y_{\nu, 1}=0, \quad \mathbf{D} Y_{\nu, 1}=1,
$$

u $n$ p $n \geqslant \lambda$

$$
L_{1}(X) \nu^{-\delta / 2}(1-4 \nu) \leqslant L_{0}\left(Y_{\nu, 1}\right)=\mathbf{E}\left|Y_{\nu, 1}\right|^{2+\delta} \leqslant L_{1}(X) \nu^{-\delta / 2}(1+40 \nu) .
$$

Если же $F \in \mathscr{F}_{2+\delta, \mathrm{s}}$, mo $u Y_{\nu, 1} \in \mathscr{F}_{2+\delta, \mathrm{s}}$.

Д о к а $з$ а т е л ь с т в о. Все утверждения леммы, за исключением нижней оценки для $\mathbf{E}\left|Y_{\nu, 1}\right|^{2+\delta}$ и последнего предложения, доказаны в [13] (также см. [5]). Для доказательства оставшихся утверждений заметим, что в силу безграничной делимости обобщенного пуассоновского распределения случайная величина $Y_{\nu, 1}$ представима в виде

$$
Y_{\nu, 1} \stackrel{d}{=} \frac{X_{1}+\cdots+X_{N_{\nu}}-\nu \mathbf{E} X}{\sqrt{\nu \mathbf{E} X^{2}}} \equiv \frac{S_{\nu}-\mathbf{E} S_{\nu}}{\sqrt{\mathbf{D} S_{\nu}}} .
$$

Отсюда непосредственно вытекает, что если распределение случайной величины $X_{1}\left(\stackrel{d}{=} X_{2} \stackrel{d}{=} \cdots \stackrel{d}{=} X\right)$ симметрично, то $\mathbf{E} X=0$ и распределение их случайной суммы $Y_{\nu, 1}$ тоже симметрично.

Обозначим теперь $a=\mathbf{E} X$. По формуле полной вероятности и в силу неравенства $e^{-x} \geqslant 1-x, x \in \mathbf{R}$, имеем

$$
\begin{aligned}
& \left(\nu \mathbf{E} X^{2}\right)^{1+\delta / 2} \mathbf{E}\left|Y_{\nu, 1}\right|^{2+\delta}=\mathbf{E}\left|X_{1}+\cdots+X_{N_{\nu}}-a \nu\right|^{2+\delta} \\
& \quad=\sum_{k=0}^{\infty} \mathbf{E}\left|X_{1}+\cdots+X_{k}-a \nu\right|^{2+\delta} \mathbf{P}\left(N_{\nu}=k\right) \\
& \quad=e^{-\nu}\left(|a \nu|^{2+\delta}+\nu \mathbf{E}\left|X_{1}-a \nu\right|^{2+\delta}+\sum_{k=2}^{\infty} \frac{\nu^{k}}{k !} \mathbf{E}\left|X_{1}+\cdots+X_{k}-a \nu\right|^{2+\delta}\right) \\
& \quad \geqslant \nu(1-\nu) \mathbf{E}\left|X_{1}-a \nu\right|^{2+\delta} .
\end{aligned}
$$

Далее, используя неравенство $|1-x|^{\alpha} \geqslant 1-\alpha x$, справедливое для всех $x \in \mathbf{R}$ и $\alpha \geqslant 1$, получаем

$$
\begin{aligned}
& \mathbf{E}\left|X_{1}-a \nu\right|^{2+\delta}=\mathbf{E}|X-a \nu|^{2+\delta} \geqslant \mathbf{E}|X-a \nu|^{2+\delta} \mathbf{1}(X \neq 0) \\
& \quad=\mathbf{E}|X|^{2+\delta}\left|1-\frac{a \nu}{X}\right|^{2+\delta} \mathbf{1}(X \neq 0) \geqslant \mathbf{E}|X|^{2+\delta}\left(1-(2+\delta) \frac{a \nu}{X}\right) \mathbf{1}(X \neq 0) \\
& \quad=\mathbf{E}|X|^{2+\delta}-(2+\delta) \nu a \mathbf{E} X|X|^{\delta}
\end{aligned}
$$

Поскольку в силу неравенства Ляпунова

$$
\left.\left.|a \mathbf{E} X| X\right|^{\delta}|=| \mathbf{E} X \cdot \mathbf{E} X|X|^{\delta}|\leqslant \mathbf{E}| X|\cdot \mathbf{E}| X\right|^{1+\delta} \leqslant \mathbf{E}|X|^{2+\delta}=\beta_{2+\delta},
$$


приходим к оценке

$$
\mathbf{E}\left|X_{1}-a \nu\right|^{2+\delta} \geqslant \beta_{2+\delta}(1-(2+\delta) \nu) \geqslant \beta_{2+\delta}(1-3 \nu),
$$

откуда окончательно получаем

$$
\begin{aligned}
\mathbf{E}\left|Y_{\nu, 1}\right|^{2+\delta} & \geqslant \frac{\nu(1-\nu) \mathbf{E}\left|X_{1}-a \nu\right|^{2+\delta}}{\left(\nu \mathbf{E} X^{2}\right)^{1+\delta / 2}} \geqslant \frac{\beta_{2+\delta}(1-3 \nu)(1-\nu)}{\left(\mathbf{E} X^{2}\right)^{1+\delta / 2} \nu^{\delta / 2}} \\
& \geqslant \frac{\beta_{2+\delta}(1-4 \nu)}{\left(\mathbf{E} X^{2}\right)^{1+\delta / 2} \nu^{\delta / 2}}=\nu^{-\delta / 2} L_{1}(X)(1-4 \nu) .
\end{aligned}
$$

Лемма доказана.

Д ок аз а т е ль с т в о т е о ре м 1 и 2 . По лемме 2 для любых $n \geqslant \lambda>0$ имеем $\Delta_{\lambda}(X)=\Delta_{n}^{*}\left(Y_{\nu, 1}\right)$ и, поскольку $\ell_{\lambda}=L_{1}(X) \lambda^{-\delta / 2}$,

$$
\begin{aligned}
\left|\frac{L_{0}\left(Y_{\nu, 1}\right)}{n^{\delta / 2}}-\ell_{\lambda}\right| & =\ell_{\lambda}\left|\frac{\nu^{\delta / 2} \mathbf{E}\left|Y_{\nu, 1}\right|^{2+\delta}}{L_{1}(X)}-1\right| \leqslant \ell_{\lambda} \max \{40 \nu, 4 \nu\} \\
& =40 \nu \ell_{\lambda}=\frac{40 \lambda \ell_{\lambda}}{n} \equiv \theta_{n} \rightarrow 0, \quad n \rightarrow \infty,
\end{aligned}
$$

для любых фиксированных $\lambda>0$ и $\ell_{\lambda}>0$. Таким образом, в силу леммы 1 и произвольности $n \geqslant \lambda$ заключаем, что для любых фиксированных $\lambda>0$ и $X \in \mathscr{F}_{2+\delta}$ (а значит, и $\ell_{\lambda}$ тоже фиксировано)

$$
\begin{aligned}
\Delta_{\lambda}(X) & =\lim _{n \rightarrow \infty} \Delta_{n}^{*}\left(Y_{\lambda / n, 1}\right) \leqslant \limsup _{n \rightarrow \infty} \sup _{Y \in \mathscr{F}_{2+\delta}:\left|L_{0}^{2+\delta}(Y) n^{-\delta / 2}-\ell_{\lambda}\right| \leqslant \theta_{n}} \Delta_{n}^{*}(Y) \\
& \leqslant C(\delta) \ell_{\lambda}+\frac{\ell_{\lambda}^{1 / \delta}}{2 \sqrt{2 \pi}}+A_{\delta}\left(\ell_{\lambda} \wedge \ell_{*}(\delta)\right) \cdot \ell_{\lambda}^{2}
\end{aligned}
$$

с определенной выше бесконечно малой последовательностью $\theta_{n}$. Если же $X \in \mathscr{F}_{2+\delta, \mathrm{s}}$, то, как отмечено в лемме 2 , и $Y_{\nu, 1} \in \mathscr{F}_{2+\delta, \mathrm{s}}$, и при всех $\lambda>0$

$$
\begin{aligned}
\Delta_{\lambda}(X) & =\lim _{n \rightarrow \infty} \Delta_{n}^{*}\left(Y_{\lambda / n, 1}\right) \leqslant \limsup _{n \rightarrow \infty} \sup _{Y \in \mathscr{F}_{2+\delta, s}:\left|L_{0}^{+\delta}(Y) n^{-\delta / 2}-\ell_{\lambda}\right| \leqslant \theta_{n}} \Delta_{n}^{*}(Y) \\
& \leqslant C_{\mathrm{s}}(\delta) \ell_{\lambda}+\frac{\ell_{\lambda}^{1 / \delta}}{2 \sqrt{2 \pi}}+A_{\delta}^{\mathrm{s}}\left(\ell_{\lambda} \wedge \ell_{*}^{\mathrm{s}}(\delta)\right) \cdot \ell_{\lambda}^{2},
\end{aligned}
$$

с той же бесконечно малой последовательностью $\theta_{n}$.

\section{3. Нижние оценки.}

Теорема 5. Для верхней асимптотически правильной константь $\bar{C}_{\mathrm{A \Pi}}\left(\mathscr{F}_{2+\delta}\right)$, определенной в $(4)$, при всех $0 \leqslant \delta \leqslant 1$ справедливы нижние оченки

$$
\bar{C}_{\mathrm{A} \Pi}\left(\mathscr{F}_{2+\delta}\right) \geqslant \bar{C}_{\mathrm{A} \Pi}^{*}\left(\mathscr{F}_{2+\delta}\right) \geqslant \sup _{\gamma>0, m \in \mathbf{N}_{0}} \gamma^{\delta / 2}\left(e^{-\gamma} \sum_{k=0}^{m} \frac{\gamma^{k}}{k !}-\Phi\left(\frac{m-\gamma}{\sqrt{\gamma}}\right)\right)
$$


где $\mathbf{N}_{0}-$ множество чельх неотрицательных чисел, $\gamma^{\delta / 2} \equiv 0$ при $\delta=0$,

$$
\begin{aligned}
\bar{C}_{\mathrm{A} \Pi}^{*}\left(\mathscr{F}_{2}\right) & =\bar{C}_{\mathrm{A} \Pi\left(\mathscr{F}_{2}\right),} \\
\bar{C}_{\mathrm{A} \Pi}^{*}\left(\mathscr{F}_{2+\delta}\right) & =\sup _{\ell>0} \limsup _{\lambda \rightarrow \infty} \sup _{F \in \mathscr{F}_{2+\delta}: \ell_{\lambda}(F)=\ell} \frac{\Delta_{\lambda}(F)}{\ell}, \quad 0<\delta \leqslant 1 .
\end{aligned}
$$

3 а м е ч а н и е 2. Можно убедиться, что экстремум в (13) при всех $0 \leqslant \delta \leqslant 0.95$ доставляется значением $m=0$, так что миноранта в (13) имеет вид

$$
\sup _{\gamma>0} \gamma^{\delta / 2}\left(e^{-\gamma}-\Phi(-\sqrt{\gamma})\right)
$$

При $\delta=0.96$ экстремальное $m$ уже равно 1 и значение миноранты в (13) есть $0.2635 \ldots$, при $\delta=0.995$ экстремальное $m$ равно 2 , соответствующее значение миноранты равно $0.2652 \ldots$ и т.д. При $\delta=1$ экстремум доставляют $m \rightarrow \infty$ и $\gamma=m$, так что $\bar{C}_{\text {АП }}\left(\mathscr{F}_{3}\right) \geqslant 2 /(3 \sqrt{2 \pi})>0.2659$. Значения миноранты в (13) при других $0<\delta<1$ приведены в таблице 1.

Теорема 6. Для нижней асимптотически правильной константы $\underline{C}_{\mathrm{A \Pi}}\left(\mathscr{F}_{3}\right)$ справедлива нижняя оченка

$$
\underline{C_{\mathrm{A \Pi}}}\left(\mathscr{F}_{3}\right) \equiv \limsup _{\ell \rightarrow 0} \limsup _{\lambda \rightarrow \infty} \sup _{F \in \mathscr{F}_{3}: \ell_{\lambda}(F)=\ell} \frac{\Delta_{\lambda}(F)}{\ell} \geqslant \frac{2}{3 \sqrt{2 \pi}}=0.2659 \ldots
$$

Доказ а тельство т е о рем 5 и 6. Первое неравенство теоремы 5

$$
\bar{C}_{\text {AП }}\left(\mathscr{F}_{2+\delta}\right) \equiv \limsup _{\lambda \rightarrow \infty} \sup _{F \in \mathscr{F}_{2+\delta}} \frac{\Delta_{\lambda}(F)}{\ell_{\lambda}(F)} \geqslant \sup _{\ell>0} \limsup _{\lambda \rightarrow \infty} \sup _{F \in \mathscr{F}_{2+\delta}: \ell_{\lambda}(F)=\ell} \frac{\Delta_{\lambda}(F)}{\ell}
$$

очевидно. Рассмотрим теперь последовательность независимых одинаково распределенных случайных величин $X, X_{1}, X_{2}, \ldots$ с распределением Бернулли $\mathbf{P}(X=1)=\gamma / \lambda=1-\mathbf{P}(X=0), 0<\gamma<\lambda$. Тогда $\mathbf{E} X=\mathbf{E} X^{2}=\mathbf{E}|X|^{2+\delta}=\gamma / \lambda, \ell_{\lambda}=\gamma^{-\delta / 2}$. Из условия $\ell_{\lambda}=\ell$ находим, что $\gamma=\ell^{-2 / \delta}$, будем иметь это соотношение в виду. Случайная величина $S_{\lambda}=X_{1}+\cdots+X_{N_{\lambda}}$, как известно, имеет пуассоновское распределение с параметром $\gamma$, а следовательно, $\mathbf{E} S_{\lambda}=\gamma=\mathbf{D} S_{\lambda}$ и

$$
\begin{aligned}
\Delta_{\lambda} & =\sup _{x}\left|\mathbf{P}\left(\frac{S_{\lambda}-\gamma}{\sqrt{\gamma}} \leqslant x\right)-\Phi(x)\right| \geqslant \sup _{m \in \mathbf{N}_{0}}\left(\mathbf{P}\left(S_{\lambda} \leqslant m\right)-\Phi\left(\frac{m-\gamma}{\sqrt{\gamma}}\right)\right) \\
& =\sup _{m \in \mathbf{N}_{0}}\left(e^{-\gamma} \sum_{k=0}^{m} \frac{\gamma^{k}}{k !}-\Phi\left(\frac{m-\gamma}{\sqrt{\gamma}}\right)\right),
\end{aligned}
$$

и для выбранного распределения $\Delta_{\lambda}$ не зависит от $\lambda$. 
Таким образом, для $\bar{C}_{\text {Ап }}^{*}\left(\mathscr{F}_{2+\delta}\right)$ при всех $0 \leqslant \delta \leqslant 1$ получаем оценку

$$
\begin{aligned}
\bar{C}_{\mathrm{A \Pi}}^{*}\left(\mathscr{F}_{2+\delta}\right) & \geqslant \sup _{\gamma>0} \limsup _{\lambda \rightarrow \infty} \sup _{F \in \mathscr{F}_{2+\delta}: \ell_{\lambda}(F)=\gamma^{-\delta / 2}} \gamma^{\delta / 2} \Delta_{\lambda}(F) \\
& \geqslant \sup _{\gamma>0, m \in \mathbf{N}_{0}} \gamma^{\delta / 2}\left(e^{-\gamma} \sum_{k=0}^{m} \frac{\gamma^{k}}{k !}-\Phi\left(\frac{m-\gamma}{\sqrt{\gamma}}\right)\right),
\end{aligned}
$$

что и доказывает теорему 5 .

Для доказательства теоремы 6 заметим, что верхний предел при $\ell \rightarrow 0$ в определении $\underline{C_{\mathrm{A \Pi}}}\left(\mathscr{F}_{3}\right)$ не уменьшится, если брать его по $\ell$ вида $\gamma^{-1 / 2}$, где $\gamma \in \mathbf{N}, \gamma \rightarrow \infty$, поэтому

$$
\begin{aligned}
\underline{C_{\mathrm{A} \Pi}}\left(\mathscr{F}_{3}\right) & \geqslant \lim _{\gamma \rightarrow \infty, \gamma \in \mathbf{N}} \limsup _{\lambda \rightarrow \infty} \sup _{F \in \mathscr{F}_{3}: \ell_{\lambda}(F)=\gamma^{-1 / 2}} \sqrt{\gamma} \Delta_{\lambda}(F) \\
& \geqslant \lim _{\gamma \rightarrow \infty, \gamma \in \mathbf{N}} \sup _{m \in \mathbf{N}_{0}} \sqrt{\gamma}\left(e^{-\gamma} \sum_{k=0}^{m} \frac{\gamma^{k}}{k !}-\Phi\left(\frac{m-\gamma}{\sqrt{\gamma}}\right)\right) \\
& \geqslant \lim _{m \rightarrow \infty, m \in \mathbf{N}} \sqrt{m}\left(e^{-m} \sum_{k=0}^{m} \frac{m^{k}}{k !}-\Phi(0)\right) .
\end{aligned}
$$

Как известно, (см., например, [1, пп. 6.5.13, 6.1.38, 6.5.35]),

$$
\begin{gathered}
e^{-m} \sum_{k=0}^{m} \frac{m^{k}}{k !}=\frac{1}{m !} \int_{m}^{\infty} t^{m} e^{-t} d t=\frac{\Gamma(m+1, m)}{m !}, \\
m !=\sqrt{2 \pi} m^{m+1 / 2} \exp \left\{-m+\frac{\theta}{12 m}\right\}, \quad 0<\theta<1, \\
\Gamma(m+1, m)=\left(\frac{m}{e}\right)^{m}\left(\sqrt{\frac{\pi m}{2}}+\frac{2}{3}+O\left(\frac{1}{\sqrt{m}}\right)\right), \quad m \rightarrow \infty,
\end{gathered}
$$

поэтому

$$
\underline{C_{\mathrm{A} \Pi}}\left(\mathscr{F}_{3}\right) \geqslant \lim _{m \rightarrow \infty, m \in \mathbf{N}} \sqrt{m}\left(\frac{1}{2}+\frac{2}{3 \sqrt{2 \pi m}}+O\left(\frac{1}{m}\right)-\Phi(0)\right)=\frac{2}{3 \sqrt{2 \pi}},
$$

что доказывает теорему 6 .

Из теорем 3 и 6 вытекает следующее утверждение.

Следствие 3. Для $\delta=1$ значения асимптотически правильной и нижней асимптотически правильной констант совпадают и равняются

$$
C_{\mathrm{A} \Pi}\left(\mathscr{F}_{3}\right)=\underline{C_{\mathrm{A} \Pi}}\left(\mathscr{F}_{3}\right)=\frac{2}{3 \sqrt{2 \pi}}=0.2659 \ldots
$$

3 а м е ч а н и е 3 . В отличие от рассматриваемой в данной работе схемы пуассоновского случайного суммирования для схемы суммирования детерминированного числа независимых одинаково распределенных случайных величин из $\mathscr{F}_{3}$ асимптотически правильная константа $(\sqrt{10}+3) /(6 \sqrt{2 \pi})=0.4097 \ldots$ строго больше нижней асимптотически правильной константы $2 /(3 \sqrt{2 \pi})=0.2659 \ldots$. 
Теорема 7. Для верхней асимптотически правильной константь $\bar{C}_{\mathrm{A \Pi}}\left(\mathscr{F}_{2, \mathrm{~s}}\right)$ справедлива оченка

$$
\bar{C}_{\mathrm{A} \Pi}\left(\mathscr{F}_{2, \mathrm{~s}}\right) \equiv \limsup _{\lambda \rightarrow \infty} \sup _{F \in \mathscr{F}_{2, \mathrm{~s}}} \Delta_{\lambda}(F) \geqslant \frac{1}{2}
$$

Д о к а з а т е л ь с т в о. Рассмотрим последовательность независимых случайных величин $X, X_{1}, X_{2}, \ldots$ с одинаковым симметричным распределением $\mathbf{P}(X= \pm 1)=p, \mathbf{P}(X=0)=1-2 p, 0<p \leqslant 1 / 2$. Тогда $\mathbf{E} X=0, \mathbf{E} X^{2}=2 p$. В [9, лемма 2.1] показано, что для функции распределения $F_{\lambda}$ соответствующей нормированной суммы $\widetilde{S}_{\lambda}=$ $\left(X_{1}+\cdots+X_{N_{\lambda}}\right) / \sqrt{2 \lambda p}$ имеет место соотношение

$$
\Phi(0)-F_{\lambda}(0)=\frac{e^{-2 \lambda p}}{2} \sum_{k=0}^{\infty} \frac{(\lambda p)^{2 k}}{(k !)^{2}},
$$

с помощью которого получаем

$$
\bar{C}_{\mathrm{A \Pi}}\left(\mathscr{F}_{2, \mathrm{~s}}\right) \geqslant \limsup _{\lambda \rightarrow \infty} \sup _{0<p \leqslant 1 / 2} \frac{e^{-2 \lambda p}}{2} \sum_{k=0}^{\infty} \frac{(\lambda p)^{2 k}}{(k !)^{2}} .
$$

Полагая теперь $p=\gamma /(2 \lambda), 0<\gamma \leqslant \lambda$, окончательно получаем

$\bar{C}_{\mathrm{A} \Pi}\left(\mathscr{F}_{2, \mathrm{~s}}\right) \geqslant \sup _{\gamma>0} \limsup _{\lambda \rightarrow \infty} \frac{e^{-\gamma}}{2} \sum_{k=0}^{\infty} \frac{(\gamma / 2)^{2 k}}{(k !)^{2}} \geqslant \frac{1}{2} \lim _{\gamma \rightarrow 0} e^{-\gamma}\left(1+\sum_{k=1}^{\infty} \frac{(\gamma / 2)^{2 k}}{(k !)^{2}}\right)=\frac{1}{2}$,

что и требовалось доказать.

Теорема 8. Пусть $\psi(\lambda), \lambda>0$, - неотрицательная функиия та$\kappa a я$, что $\psi(\lambda) \rightarrow 0$ при $\lambda \rightarrow \infty$. Тогда найдутся такие функиии распределения $F \in \mathscr{F}_{2}$ и положительное число $\lambda_{1}$, что $\Delta_{\lambda}(F) \geqslant \psi(\lambda)$ при всех $\lambda \geqslant \lambda_{1}$. При этом функиия распределения $F$ имеет вид

$$
F(x)=F\left(x ; \gamma_{1}, \gamma_{2}, \ldots ; \sigma_{1}, \sigma_{2}, \ldots\right)=\sum_{k=1}^{\infty} \gamma_{k} \Phi\left(\frac{x}{\sigma_{k}}\right),
$$

где $\gamma_{k} \geqslant 0, \sigma_{k}>0, k=1,2, \ldots, \sum_{k=1}^{\infty} \gamma_{k}=1, \sum_{k=1}^{\infty} \gamma_{k} \sigma_{k}^{2}=1$.

3 а м е ч а н и е 4. При доказательстве теоремы числовые последовательности $\left\{\gamma_{k}\right\}_{k \geqslant 1}$ и $\left\{\sigma_{k}\right\}_{k \geqslant 1}$, определяющие экстремальную функцию распределения $F$ в (14), будут построены по функции $\psi(\lambda)$ в явном виде.

Основные идеи доказательства восходят к методу ПрохороваМацкявичюса, с помощью которого в [8] был получен аналогичный результат для сумм детерминированного числа независимых одинаково распределенных случайных величин. Как и в [8], мы разобьем доказательство теоремы 8 на два этапа: сначала выведем неравенство, связывающее величину $\Delta_{\lambda}(F)$ с остатками рядов

$$
r_{n}=\sum_{k=n+1}^{\infty} \gamma_{k}, \quad R_{n}=\sum_{k=n+1}^{\infty} \gamma_{k} \sigma_{k}^{2}, \quad n=0,1,2, \ldots
$$


а затем перейдем к построению функции распределения $F$ вида $(14)$, существование которой утверждает теорема 8. Доказательство теоремы 8 исправляет неточность, допущенную Мацкявичюсом при доказательстве аналогичной теоремы в [8]: доказательство Мацкявичюса верно при условии, что последовательность $\psi(n)$ строго монотонна.

Лемма 3. Для любых $\lambda \geqslant 1$ u $n \geqslant 0$ таких, что $0<4 r_{n}(\lambda+1) \leqslant$ $R_{n} \leqslant 1$, справедлива оченка

$$
\Delta_{\lambda}>\frac{R_{n}}{60}
$$

Д о к а з а т е л ь с т в о. Как несложно убедиться, характеристическая функция распределения (14) имеет вид

$$
f(t)=\sum_{k=1}^{\infty} \gamma_{k} \exp \left\{-\frac{\sigma_{k}^{2} t^{2}}{2}\right\}, \quad t \in \mathbf{R}
$$

а характеристическая функция нормированной суммы $\widetilde{S}_{\lambda}=\left(X_{1}+\cdots+\right.$ $\left.X_{N_{\lambda}}\right) / \sqrt{\lambda}$ имеет вид

$$
f_{\lambda}(t)=\exp \left\{\lambda\left(f\left(\frac{t}{\sqrt{\lambda}}\right)-1\right)\right\}=\exp \left\{\lambda \sum_{k=1}^{\infty} \gamma_{k}\left(\exp \left\{-\frac{\sigma_{k}^{2} t^{2}}{2 \lambda}\right\}-1\right)\right\} .
$$

В силу неравенства $e^{x} \geqslant 1+x$, справедливого для всех $x \in \mathbf{R}$, имеем

$$
\begin{aligned}
f_{\lambda}(t)-e^{-t^{2} / 2} & =e^{-t^{2} / 2}\left(f_{\lambda}(t) e^{t^{2} / 2}-1\right) \\
& =e^{-t^{2} / 2}\left[\exp \left\{\lambda \sum_{k=1}^{\infty} \gamma_{k}\left(\exp \left\{-\frac{\sigma_{k}^{2} t^{2}}{2 \lambda}\right\}-1+\frac{t^{2}}{2 \lambda}\right)\right\}-1\right] \\
& \geqslant \lambda e^{-t^{2} / 2} \sum_{k=1}^{\infty} \gamma_{k}\left(\exp \left\{-\frac{\sigma_{k}^{2} t^{2}}{2 \lambda}\right\}-1+\frac{t^{2}}{2 \lambda}\right) \geqslant 0 .
\end{aligned}
$$

Далее, как показано в [8], для любой пары функций распределения $G_{1}$ и $G_{2}$ с характеристическими функциями $g_{1}$ и $g_{2}$ справедлива оценка

$$
\sup _{x \in \mathbf{R}}\left|G_{1}(x)-G_{2}(x)\right| \geqslant \frac{1}{2 \sqrt{2 \pi}}\left|\int_{-\infty}^{\infty}\left(g_{1}(t)-g_{2}(t)\right) e^{-t^{2} / 2} d t\right| .
$$

В частности, для $G_{1}=F_{\lambda}$ и $G_{2}=\Phi$ имеем

$$
\begin{aligned}
\Delta_{\lambda} & \geqslant \frac{1}{2 \sqrt{2 \pi}} \int_{-\infty}^{\infty}\left(f_{\lambda}(t)-e^{-t^{2} / 2}\right) e^{-t^{2} / 2} d t \\
& \geqslant \frac{\lambda}{2 \sqrt{2 \pi}} \sum_{k=1}^{\infty} \gamma_{k} \int_{-\infty}^{\infty}\left(\exp \left\{-\frac{\sigma_{k}^{2} t^{2}}{2 \lambda}\right\}-1+\frac{t^{2}}{2 \lambda}\right) e^{-t^{2}} d t \\
& =\frac{\lambda}{2 \sqrt{2}}\left(\sum_{k=1}^{\infty} \gamma_{k}\left(1+\frac{\sigma_{k}^{2}}{2 \lambda}\right)^{-1 / 2}-1+\frac{1}{4 \lambda}\right),
\end{aligned}
$$


так как

$$
\begin{gathered}
\int_{-\infty}^{\infty} \exp \left\{-\frac{\sigma^{2} t^{2}}{2 \lambda}-t^{2}\right\} d t=\sqrt{2 \pi}\left(1+\frac{\sigma^{2}}{2 \lambda}\right)^{-1 / 2} \\
\int_{-\infty}^{\infty} e^{-t^{2}} d t=\sqrt{\pi}, \quad \int_{-\infty}^{\infty} t^{2} e^{-t^{2}} d t=\frac{\sqrt{\pi}}{2}
\end{gathered}
$$

Так как функция $1 / \sqrt{x}$ выпукла при $x>0$, то по неравенству Иенсена для всех $n$ таких, что $\sum_{k=1}^{n} \gamma_{k} \equiv 1-r_{n}>0$, получаем

$$
\begin{aligned}
& \sum_{k=1}^{n} \gamma_{k}\left(1+\frac{\sigma_{k}^{2}}{2 \lambda}\right)^{-1 / 2}=\left(1-r_{n}\right) \sum_{k=1}^{n} \frac{\gamma_{k}}{1-r_{n}}\left(1+\frac{\sigma_{k}^{2}}{2 \lambda}\right)^{-1 / 2} \\
& \quad \geqslant\left(1-r_{n}\right)\left(\sum_{k=1}^{n} \frac{\gamma_{k}}{1-r_{n}}\left(1+\frac{\sigma_{k}^{2}}{2 \lambda}\right)\right)^{-1 / 2}=\left(1-r_{n}\right)\left(1+\frac{1-R_{n}}{2 \lambda\left(1-r_{n}\right)}\right)^{-1 / 2}
\end{aligned}
$$

Аналогично, для всех $n$ таких, что $r_{n}>0$,

$$
\sum_{k=n+1}^{\infty} \gamma_{k}\left(1+\frac{\sigma_{k}^{2}}{2 \lambda}\right)^{-1 / 2} \geqslant r_{n}\left(1+\frac{R_{n}}{2 \lambda r_{n}}\right)^{-1 / 2}
$$

Таким образом, при всех $n$ таких, что $r_{n} \in(0,1)$, справедлива оценка

$$
\Delta_{\lambda} \geqslant \frac{\lambda}{2 \sqrt{2}} \Delta_{\lambda}^{*}\left(r_{n}, R_{n}\right)
$$

где

$$
\Delta_{\lambda}^{*}(r, R)=(1-r)\left(1+\frac{1-R}{2 \lambda(1-r)}\right)^{-1 / 2}+r\left(1+\frac{R}{2 \lambda r}\right)^{-1 / 2}-1+\frac{1}{4 \lambda}
$$

Зафиксируем $r \in(0,1)$ и рассмотрим $\Delta_{\lambda}^{*}$ как функцию переменного $R \in$ $[r, 1]$. Оценим $\Delta_{\lambda}^{*}(r, R)$ на указанном отрезке снизу. Несложно убедиться, что

$$
\begin{gathered}
\Delta_{\lambda}^{*}(r, r)=\left(1+\frac{1}{2 \lambda}\right)^{-1 / 2}-1+\frac{1}{4 \lambda}>0 \quad \text { при всех } \lambda>0, \\
\frac{\partial \Delta_{\lambda}^{*}(r, R)}{\partial R}=\frac{A^{-3 / 2}-B^{-3 / 2}}{4 \lambda}, \quad \text { где } A=1+\frac{1-R}{2 \lambda(1-r)}, \quad B=1+\frac{R}{2 \lambda r} .
\end{gathered}
$$

Далее, в рассматриваемой области $R \in[r, 1]$ при всех $\lambda \geqslant 1$ справедливы неравенства $A \leqslant B$ и $A \leqslant 1+(2 \lambda)^{-1} \leqslant 3 / 2$, а следовательно,

$$
A^{-3 / 2}-B^{-3 / 2} \geqslant \frac{B-A}{A^{3 / 2} B}=\frac{A^{-3 / 2}}{(1-r)} \frac{V}{1+V}, \quad \text { где } V=V(R)=\frac{R-r}{(2 \lambda+1) r} .
$$


Выбирая теперь произвольное $R_{*} \in(r, 1]$ и обозначая $V_{*}=V\left(R_{*}\right)$, получаем

$$
\begin{aligned}
\Delta_{\lambda}^{*}\left(r, R_{*}\right)=\Delta_{\lambda}^{*}(r, r)+\int_{r}^{R_{*}} \frac{\partial \Delta_{\lambda}^{*}(r, R)}{\partial R} d R \geqslant \int_{r}^{R_{*}} \frac{\partial \Delta_{\lambda}^{*}(r, R)}{\partial R} d R \\
\quad \geqslant \frac{A^{-3 / 2}}{4 \lambda(1-r)} \int_{r}^{R_{*}} \frac{V(R) d R}{1+V(R)}=\frac{(2 \lambda+1) r A^{-3 / 2}}{4 \lambda(1-r)} \int_{0}^{V_{*}} \frac{V d V}{1+V} \\
\quad=\frac{(2 \lambda+1) r A^{-3 / 2}}{4 \lambda(1-r)}\left(V_{*}-\ln \left(1+V_{*}\right)\right)=\frac{\left(R_{*}-r\right) A^{-3 / 2}}{4 \lambda(1-r)} \frac{V_{*}-\ln \left(1+V_{*}\right)}{V_{*}} \\
\quad \geqslant \frac{(3 / 2)^{-3 / 2}\left(R_{*}-r\right)}{4 \lambda} \frac{V_{*}-\ln \left(1+V_{*}\right)}{V_{*}} .
\end{aligned}
$$

Предположим, что $r \leqslant(4(\lambda+1))^{-1}$. Тогда для любого $R_{*}$ из области $4 r(\lambda+1) \leqslant R_{*} \leqslant 1$ при всех $\lambda \geqslant 1$

$$
\begin{gathered}
R_{*}-r \geqslant R_{*}-\frac{R_{*}}{4(\lambda+1)}=R_{*}\left(1-\frac{1}{4(\lambda+1)}\right) \geqslant \frac{7 R_{*}}{8}, \\
V_{*}=\frac{R_{*}-r}{(2 \lambda+1) r} \geqslant \frac{4 r(\lambda+1)-r}{(2 \lambda+1) r}=\frac{4 \lambda+3}{2 \lambda+1}>2 .
\end{gathered}
$$

Так как функция $(V-\ln (1+V)) / V$ возрастает при $V>0$, то ее значение в точке $V_{*}$ больше, чем ее значение в точке 2 , т.е. больше, чем $1-\ln \sqrt{3}=$ $0.4506 \ldots$. Поэтому при всех $\lambda \geqslant 1, r>0$ и $R_{*}$ таких, что $4 r(\lambda+1) \leqslant$ $R_{*} \leqslant 1$, имеем

$$
\Delta_{\lambda}^{*}\left(r, R_{*}\right) \geqslant \frac{(3 / 2)^{-3 / 2}}{4 \lambda} \cdot \frac{7}{8} \cdot(1-\ln \sqrt{3}) R_{*} .
$$

Итак, если выполнено условие леммы, т.е. если $\lambda \geqslant 1$ и $n$ таковы, что $0<4 r_{n}(\lambda+1) \leqslant R_{n} \leqslant 1$, то

$$
\begin{aligned}
\Delta_{\lambda} & \geqslant \frac{\lambda}{2 \sqrt{2}} \cdot \frac{(3 / 2)^{-3 / 2}}{4 \lambda} \cdot \frac{7}{8} \cdot(1-\ln \sqrt{3}) R_{n} \\
& =\frac{7 \sqrt{3}}{4 \cdot 8 \cdot 9}(1-\ln \sqrt{3}) R_{n} \geqslant 0.0189 \cdot R_{n}>\frac{1}{60} R_{n} .
\end{aligned}
$$

Лемма доказана.

Доказательст в о те о ремы 8. Для того чтобы иметь дело с монотонными функциями, положим $\psi_{1}(\lambda)=\sup _{x \geqslant \lambda} \psi(x)$. Тогда $\psi_{1}(\lambda)$ не возрастает и $\lim _{\lambda \rightarrow \infty} \psi_{1}(\lambda)=0$. Без ограничения общности будем считать, что $\psi_{1}(\lambda)$ положительна при всех $\lambda>0$, так как в противном случае $\psi_{1}(\lambda)=\psi(\lambda) \equiv 0$ для всех $\lambda$ начиная с некоторого и утверждение теоремы становится тривиальным. Определим $n_{0}$ как такое наименьшее целое положительное число, что выполняется неравенство $60 \psi_{1}\left(n_{0}\right) \leqslant 1$. Положим

$$
R_{0}=\cdots=R_{n_{0}-1}=1, \quad R_{n_{0}+k}=60 \psi_{1}\left(n_{0}+k\right), \quad k \geqslant 0,
$$


T.e.

$$
\begin{gathered}
\gamma_{1}=\gamma_{2}=\cdots=\gamma_{n_{0}-1}=0, \quad \gamma_{n_{0}} \sigma_{n_{0}}^{2}=1-60 \psi_{1}\left(n_{0}\right) \\
\gamma_{n_{0}+k} \sigma_{n_{0}+k}^{2}=60\left(\psi_{1}\left(n_{0}+k-1\right)-\psi_{1}\left(n_{0}+k\right)\right), \quad k=1,2, \ldots
\end{gathered}
$$

Определим теперь $\gamma_{n_{0}+k}=r_{n_{0}+k-1}-r_{n_{0}+k}, k=0,1,2, \ldots$, где

$$
r_{n_{0}-1}=1, \quad r_{n_{0}+k}=\frac{R_{n_{0}+k}}{4(\lambda+1)}=\frac{60 \psi_{1}\left(n_{0}+k\right)}{4(\lambda+1)}, \quad k=0,1,2, \ldots
$$

Ясно, что $\sum_{k=1}^{\infty} \gamma_{k} \sigma_{k}^{2}=1, \sum_{k=1}^{\infty} \gamma_{k}=1$, и условие $4(\lambda+1) r_{n} \leqslant R_{n}$ леммы 3 выполняется очевидным образом для всех $n \geqslant n_{0}$. Поэтому в силу леммы 3 заключаем, что $\Delta_{\lambda} \geqslant R_{n} / 60=\psi_{1}(n)$ для всех $n \geqslant n_{0}$. $\mathrm{B}$ частности, если $\lambda \geqslant n_{0}$, то для $n=[\lambda]$, где $[\lambda]$ - наибольшее целое, не превосходящее $\lambda$, имеем $n \geqslant n_{0}$, а следовательно, $\Delta_{\lambda} \geqslant \psi_{1}([\lambda])$. Так как $[\lambda] \leqslant \lambda$ и $\psi_{1}(\lambda)$ не возрастает, заключаем, что $\Delta_{\lambda} \geqslant \psi_{1}(\lambda) \geqslant \psi(\lambda)$ для всех $\lambda \geqslant n_{0}$, что и требовалось доказать.

В заключение автор выражает свою искреннюю признательность В. Ю. Королеву за постоянное внимание к работе.

\section{СПИСОК ЛИТЕРАТУРЫ}

1. Абрамовии М., Стиган И. Справочник по специальным функциям. - М.: Наука, 1979,832 c.

2. Бхаттачария Р. Н., Ранга Рао Р. Аппроксимация нормальным распределением. М.: Наука, 1982, 286 с.

3. Григорьева М.Е., Шевцова И.Г. Уточнение неравенства Каца-Берри-Эссеена. - Информ. и ее примен., 2010, т. 4, № 2, с. 78-85.

4. Колмогоров A. Н. Некоторые работы последних лет в области предельных теорем теории вероятностей. - Вестн. Моск. ун-та, 1953, т. 10, с. 29-38.

5. Королев В.Ю., Бенинг В.Е., Шоргин С.Я. Математические основы теории риска. М.: Физматлит, 2011, 620 с.

6. Королев В. Ю., Шевцова И. Г. Уточнение верхней оценки абсолютной постоянной в неравенстве Берри-Эссеена для смешанных пуассоновских случайных сумм. Докл. РАН, 2010, т. 431, № 1, с. 16-19.

7. Круглов В.М., Королев В.Ю. Предельные теоремы для случайных сумм. М.: Изд-во МГУ, 1990, 296 с.

8. Мацкявичюс В. К. О нижней оценке скорости сходимости в центральной предельной теореме. - Теория вероятн. и ее примен., 1983, т. 28 , в. 3, с. 565-569.

9. Нефедова Ю. С. Уточнение структуры моментных оценок скорости сходимости в предельных теоремах для сумм независимых случайных величин. Дисс. . . . канд. физ.-матем. наук. М.: МГУ, 2011.

10. Нефедова Ю. С., Шевцова И. Г. О точности нормальной аппроксимации для распределений пуассоновских случайных сумм. - Информ. и ее примен., 2011, т. 5 , № 1 , c. $39-45$.

11. Ротарь Г.В. Некоторые задачи планирования резерва. Дисс. ... канд. физ.матем. наук. М.: ЦЭМИ, 1972.

12. Ротарь Г. В. Об одной задаче управления резервами. - Эконом. матем. методы, 1976, т. 12, № 4, с. 733-739. 
13. Шевцова И. Г. О точности нормальной аппроксимации для распределений пуассоновских случайных сумм. - Обозрение прикл. и промышл. матем., 2007, т. 14, № 1, c. $3-28$.

14. Шевцова И.Г. Нижняя асимптотически правильная постоянная в центральной предельной теореме. - Докл. РАН, 2010, т. 430, № 4, с. 466-469.

15. Шевцова И. Г. Об асимптотически правильных постоянных в неравенстве БерриЭссеена-Каца. - Теория вероятн. и ее примен., 2010, т. 55, в. 2, с. 271-304.

16. Шевцова И.Г. О точности нормальной аппроксимации для сумм независимых случайных величин. - Докл. РАН, 2012, т. 443, № 5, с. 555-560.

17. Шевцова И.Г. О точности нормальной аппроксимации для сумм независимых симметричных случайных величин. - Докл. РАН, 2012, т. 443, № 6, с. 671-676.

18. Шевцова И.Г. Моментные оценки точности нормальной аппроксимации с уточненной структурой для сумм независимых симметричных случайных величин. Теория вероятн. и ее примен., 2012, т. 57, в. 3, с. 499-532.

19. Шиганов И. С. Об уточнении верхней константы в остаточном члене центральной предельной теоремы. - Проблемы устойчивости стохастических моделей. М.: ВНИИСИ, 1982, с. 109-115.

20. van Beek P. An application of Fourier methods to the problem of sharpening the Berry-Esseen inequality. - Z. Wahrscheilichkeistheor. verw. Geb., 1972, v. 23, p. 187196.

21. Bening V. E., Korolev V. Yu. Generalized Poisson Models and Their Applications in Insurance and Finance. Utrecht: VSP, 2002, 434 p.

22. Bening V.E., Korolev V. Yu., Shorgin S. Ya. On approximation to generalized Poisson distribuions. - J. Math. Sci., 1997, v. 83, № 3, p. 360-373.

23. von Chossy R., Rappl G. Some approximation methods for the distribution of random sums. - Insurance: Math. Econ., 1983, v. 2, p. 251-270.

24. Englund G. A remainder term estimate in a random-sum central limit theorem. Теория вероятн. и ее примен., 1983, т. 28, в. 1, с. 143-149.

25. Esseen C.-G. A moment inequality with an application to the central limit theorem. Skand. Aktuarietidskr., 1956, v. 39, p. 160-170.

26. Gnedenko B. V., Korolev V. Yu. Random Summation: Limit Theorems and Applications. Boca Raton: CRC Press, 1996, 267 p.

27. Korolev V., Shevtsova I. An improvement of the Berry-Esseen inequality with applications to Poisson and mixed Poisson random sums. - Scand. Actuarial J., 2012, v. 2012 , № 2 , p. 81-105.

28. Korolev V. Yu., Shorgin S. Ya. On the absolute constant in the remainder term estimate in the central limit theorem for Poisson random sums. - Probabilistic Methods in Discrete Mathematics (Proceeding of the Fourth International Petrozavodsk Conference). Utrecht: VSP, 1997, p. 305-308.

29. Michel R. On Berry-Esseen results for the compound Poisson distribution. - Insurance: Math. Econ., 1993, v. 13, № 1, p. 35-37.

30. Nefedova Yu., Shevtsova I. On the constants in the uniform and non-uniform versions of the Berry-Esseen inequality for Poisson random sums. - Ultra Modern Telecommunications and Control Systems and Workshops (ICUMT) on 2010 International Congress (18-20 Oct. 2010, Moscow), p. 1141-1144.

31. Shevtsova I. On the absolute constants in the Berry-Esseen type inequalities for identically distributed summands. - arXiv:1111.6554v1 [math.PR].

32. Shevtsova I. Moment-type estimates with asymptotically optimal structure for the accuracy of the normal approximation. - Ann. Math. Inform., 2012, v. 39, p. 241307. 